\title{
Estudio sobre las dificultades que tienen estudiantes de nivel superior en la resolución de problemas de cálculo, y empleo de un Escenario de aprendizaje tecnopedagógico como apoyo en el trabajo del aula Study about the difficulties that higher-level students have when solving Calculus problems and the use of the technological learning scenario as a support in the classroom work
}

Elena F. Ruiz Ledesma ${ }^{1}$

Palabras clave: aplicaciones de cálculo; proceso de enseñanza; proceso de aprendizaje; recursos multimedia; resolución de problemas Keywords: calculus applications; teaching process; learning process; multimedia resources; problem solving

Recibido en: 10-02-2019 / Aceptado en: 27-05-2019

\section{Resumen}

Introducción: Se describe el trabajo llevado a cabo mediante el empleo de un escenario tecnopedagógico, el cual está conformado por el contenido de la asignatura de Cálculo Aplicado en el Nivel Superior, el uso de estrategias de enseñanza y aprendizaje (trabajo pedagógico) y el empleo de tecnología. De forma específica, se trabajó con un Sistema experto denominado SIMAATCA, en el cual hay varios recursos multimedia al que los estudiantes tienen acceso. Se pretende determinar si con el empleo del escenario tecnopedagógico los estudiantes logran mejorar su rendimiento en una de las unidades temáticas (Unidad 1. Aplicaciones de la Derivada) de la materia de Cálculo Aplicado.

Método: Se trabajó con dos grupos de 33 estudiantes cada uno, quienes se encontraban iniciando su segundo semestre en la licenciatura, un grupo fue considerado de control (GC) y el otro experimental (GE). En ambos grupos se abordó la misma Unidad temática de la asignatura, lo que diferencia a los dos grupos es la estrategia de enseñanza empleada. En el grupo de control se trabajó de forma tradicional, empleando de forma exclusiva el pizarrón y el libro de texto; mientras que en el grupo experimental se trabajó con el escenario tecnopedagógico. El rendimiento académico del estudiante se midió comparando los resultados obtenidos entre el pretest y el post-test, ambos cuestionarios aplicados a los dos grupos (de control y experimental).

Resultados: Los resultados obtenidos en el cuestionario diagnóstico (pre-test) tanto por el grupo de control (GC) como del grupo experimental (GE) fueron muy bajos, ya que los estudiantes presentaron dificultades en los planteamientos de la mayoría de los problemas. Después de haber

\footnotetext{
${ }^{1}$ Instituto Politécnico Nacional. E-mail: elenfruiz65@gmail.com

(C) Universidad De La Salle Bajío (México)
} 
trabajado con ambos grupos, empleando estrategias diferentes, se aplicó el pos-test. En esta ocasión hubo una mejora en los resultados obtenidos por ambos grupos, pero el GE sobresalió en la cantidad de respuestas correctas y la forma de enfrentar cada problema, comparado con el GC. En el grupo experimental los problemas que fueron resueltos de forma correcta por una mayor cantidad de estudiantes (97\%), correspondieron a la obtención de puntos críticos (máximos, mínimos), intervalos donde la función es creciente y en donde es decreciente, así como lo concerniente a los temas de optimización (72\% en el primer problema y $69 \%$ en el segundo) y en el problema de razón de cambio (63\%). El problema que fue resuelto de forma correcta por menos estudiantes (42\%) fue del tema de diferenciales.

Discusión o Conclusión: Se concluye que el grupo experimental mejoró su rendimiento académico al aumentar su promedio (de 2.7 a 6.0), cuya mejoría se debió a la variedad de estrategias que emplearon y al uso de la tecnología como parte del Escenario de Aprendizaje Tecnopedagógico empleado. Mientras que el grupo de control no tuvo una mejora en su rendimiento (su promedio cambió de 2.6 a 3.7), y los estudiantes emplearon en la mayoría de los problemas fórmulas pero no de forma correcta.

\begin{abstract}
Introduction: The work carried out through the use of a techno-pedagogical scenario is described, which is made up of the content of the subject of Applied Calculus in the Higher Level, the use of teaching and learning strategies (pedagogical work) and the use of technology. Specifically, we worked with an expert system called SIMAATCA, in which there are several multimedia resources to which students have access. It is intended to determine if with the employment of the techno-pedagogical scenario the students manage to improve their performance in one of the units of the curriculum (Unit 1. Applications of the Derivative) of the subject of Applied Calculus.
\end{abstract}

Method: The research study was developed with two groups of 33 students each one, who were starting their second semester in the degree, one group was considered as the control one (GC) and the other was an experimental group (GE). In both groups the same Unit of the curriculum was approached, what differentiates the two groups is the teaching strategy used. In the control group was worked in a traditional way, using exclusively the blackboard and the textbook; while in the experimental group was worked with the techno-pedagogical scenario. The student's 
Estudio sobre las dificultades que tienen estudiantes de nivel superior en la resolución de problemas de cálculo, y empleo de un Escenario de aprendizaje tecnopedagógico como apoyo en el trabajo del aula

academic performance was measured by comparing the results obtained between the pretest and the posttest, both questionnaires applied to the two groups (control and experimental).

Results: The results obtained in the diagnostic questionnaire (pre-test) both by the control group (CG) and the experimental group (EG) was very low since the students presented difficulties in the approaches of most of the problems. After having worked with both groups using different strategies, the post-test was applied. This time there was an improvement in the results obtained by both groups, but the EG excelled in the number of correct answers and the way to face each problem, compared to the CG.

\section{Introducción}

Las prácticas educativas deben adaptarse a los cambios sociales, con la finalidad de acercarse a los alumnos y aprovechar las ventajas que brindan estos cambios. En la actualidad, la presencia de las tecnologías digitales se puede apreciar en todas las actividades sociales. El Instituto Nacional de Estadística y Geografía, en México y en su encuesta sobre uso de tecnología (INEGI, 2017 , p. 1) reporta que "50.6 millones de personas son usuarios de una computadora, y 71.3 millones utilizan Internet", así que involucrar estas tecnologías en el aula se convierte en una prioridad de la labor docente.

Uno de los objetivos de la Organización de las Naciones Unidas para la Educación, la Ciencia y la Cultura (UNESCO), es el de la "Educación para todos", y está apoyando para que las personas empleen sus dispositivos móviles en actividades académicas para enfrentar los retos de los diferentes contextos educativos, y sean capaces de poder trabajar contenidos académicos en diferentes formatos (UNESCO, 2013).

El aprendizaje móvil tiene que ver con la forma en cómo el estudiante logra comunicarse, descargar información, resolver actividades y trabajar con diferentes aplicaciones en cualquier lugar y momento. (UNESCO, 2013). El aprendizaje móvil también incluye el esfuerzo que implica apoyar distintas metas educativas como es el caso de la administración efectiva de los sistemas escolares y una mejor comunicación entre las escuelas y las familias.

Por otro lado, uno de los objetivos de las escuelas de nivel superior, consiste en que sus estudiantes alcancen un nivel de desarrollo eficiente para incorporarse al campo laboral, pero una problemática que se está viviendo, es que los estudiantes al ingresar al nivel superior muestran 
deficiencias en diferentes aspectos. Uno de ellos corresponde al de las matemáticas, lo que se traduce en un incompleto desarrollo de sus competencias y, consecuentemente, en tropiezos durante su trayectoria académica en las distintas asignaturas de formación básica. Como parte de los factores que desencadenan esta situación están: la falta de hábitos de estudio; las estrategias de los profesores; el uso incorrecto de la información y de los recursos obtenidos de internet; y falta de motivación y concentración de los estudiantes, por mencionar algunos.

En la asignatura de Cálculo se ha detectado la falta de significado y sentido (Cuesta, Escalante y Ruiz, 2016) que muchos de los estudiantes de nivel medio y superior tienen de los conceptos involucrados (función, límite, derivada e integral). Algunos autores de la literatura de matemática educativa lo atribuyen a que el estudio del Cálculo se restringe a una manipulación algebraica dejando a un lado las múltiples representaciones que hay (Hitt, 2014., Sánchez, García \& Llinares, 2008., Oliveros, 1999., Duval, 1998). El punto de vista de estos investigadores, es que las tareas de conectar las diferentes representaciones de un concepto como la representación numérica, algebraica o analítica y gráfica, ayudan a su comprensión.

Esta problemática, de la falta de otorgamiento de significado y sentido a los conceptos involucrados en el Cálculo, también se refleja en los estudiantes de una de las Unidades Académicas del Instituto Politécnico Nacional (ESCOM-IPN), lo que influye en que no logran enfrentar con éxito las situaciones problemáticas que se les pide resolver en la unidad de aprendizaje de Cálculo Aplicado, trayendo como consecuencia:

- Bajo aprovechamiento en la materia

- No conectar los conceptos con los de su carrera

- Alto índice de reprobación

- Falta de interés en aprender

- Deserción

Por otro lado, Artigue (2011) señala que aunque los alumnos realizan de manera más o menos mecánica algunos cálculos de derivadas, al resolver algunos problemas estándar tienen dificultades en la comprensión satisfactoria de los conceptos y métodos de pensamiento que conforman el centro del análisis matemático. 
Una de las razones principales de este problema es que los alumnos no conectan los conceptos matemáticos con actividades que realizan, y ven en ellos sólo métodos abstractos que deben aplicar cuando se les señala que lo hagan.

Hitt $(2013,2)$ al realizar un análisis de cómo se utilizan los problemas y contextos físicos en la impartición de una clase, concluye que: "Pareciera que el contexto es importante para introducir un concepto matemático, el mismo es abandonado rápidamente, proporcionado tanto en el libro de texto como en el aula, una primicia a las representaciones algebraicas sobre las otras representaciones".

Dada la importancia que ha cobrado el aprendizaje electrónico y el móvil, se decidió realizar una investigación, a través de la cual se construyó un escenario de aprendizaje tecnopedagógico, proponiendo como caso de estudio trabajar con una de las asignaturas que tiene un alto índice de reprobación o de fracaso en estudiantes de nivel superior, que es Cálculo Aplicado, en particular se desarrolló este escenario de aprendizaje con la comunidad de estudiantes que cursan la carrera de Ingeniería en sistemas computacionales, en la Escuela Superior de Cómputo en la Ciudad de México (ESCOM-IPN), durante el semestre AgostoDiciembre de 2018. Se trabajó con un Escenario de Aprendizaje Tecnopedagógico (EATP), el cual se apoyó en lo señalado en la literatura como modelo TPACK, por sus siglas en inglés: Technological Pedagogical Content Knowledge; debido a que interesó abordar la problemática de forma integral, es decir, no solo desde el punto de vista de la asignatura de Cálculo Aplicado, sino entretejida con los aspectos pedagógicos y tecnológicos.

\section{Problema de investigación}

El problema que aborda la investigación presentada en este artículo es que hay un alto índice de reprobación en las asignaturas de Cálculo y Cálculo Aplicado en las instituciones de nivel superior del IPN. Se eligió una de las unidades académicas, la Escuela Superior de Cómputo. Se solicitaron al Departamento de Control Escolar las estadísticas de los últimos cinco años y se encontró que de la materia de Cálculo se tiene el $45 \%$ de reprobación y de Cálculo Aplicado el $40 \%$. Se propuso como premisa que uno de los motivos de la reprobación de los estudiantes es el hecho de la falta de sentido que le dan al trabajo teórico visto en clase, por lo que el propósito de la investigación fue, primeramente, revisar y entender el motivo de los errores cometidos por los estudiantes al trabajar problemas que son aplicaciones del Cálculo, mediante el análisis de las 
respuestas dadas a un cuestionario diagnóstico y, después, proponer estrategias de aprendizaje que pudieran llevarse a cabo, para motivar al estudiante a abordar diferentes situaciones, construyendo un Escenario de Aprendizaje Tecnopedagógico (EATP), el cual emplea en la parte tecnológica un sistema computacional desarrollado como parte de la investigación que se muestra en el presente documento y al que se le denominó SIMAATCA, el cual contiene diversos recursos digitales educativos. Este sistema se puede trabajar tanto en la computadora de escritorio como en dispositivos móviles.

La finalidad última de la investigación es que los estudiantes puedan resolver problemas de Cálculo logrando aplicar de forma correcta los conceptos fundamentales y que los estudiantes pudieran enfrentar con éxito los exámenes a título de suficiencia (ETS) que aplica la escuela.

La elección del modelo TPACK como método de trabajo fue debido a que propone un trabajo integral en el salón de clases al considerar no solo el contenido matemático sino el aspecto pedagógico que permite llevar a cabo los diferentes momentos del proceso enseñanzaaprendizaje de los estudiantes (inicio, desarrollo, evaluación, retroalimentación), tomando en consideración teorías psicopedagógicas para el tratamiento de los contenidos, además de que se incorpora el uso de las tecnologías de información y comunicación para poder tratar dichos contenidos buscando el desarrollo de las diferentes habilidades de los estudiantes.

Existen otros métodos reportados en la literatura de matemática educativa para abordar situaciones de matemáticas en el salón de clase, como el método denominado ACODESA propuesto por Hitt (2007) que es una adaptación a un acercamiento sociocultural del aprendizaje de las matemáticas (ver Hitt, González Martín y Morasse, 2008). Con este método, el profesor no dictamina sobre lo realizado por los alumnos en las primeras etapas, salvo al final en donde se da un proceso de institucionalización. El profesor es un guía y es deber de los estudiantes el argumentar y validar sus producciones. Aunque en este método también se toma en cuenta el uso de tecnología educativa, no fue seleccionado en la investigación presentada en este artículo debido a que lo que se buscaba es que el profesor tuviera más actuación en el proceso de enseñanza-aprendizaje, al diseñar un escenario que le permitiera organizar los contenidos de la materia para ser abordados de forma integral por parte del alumno, porque el objetivo final es que los alumnos logren resolver problemas para poder enfrentar los exámenes a título de suficiencia que aplica la escuela. 
En la parte tecnológica se trabajó con SIMAATCA, que es un sistema que se adapta al estilo de aprendizaje y al nivel de dificultad del estudiante, proporcionándole recursos digitales que son acordes a los perfiles detectados. Los recursos son multimedia: van desde PDF, hasta programas con los que puede interactuar el estudiante. SIMAATCA hasta ahora contiene recursos que corresponden a los temas del plan de estudios de la Unidad de Aprendizaje de Cálculo Aplicado (ESCOM-IPN, 2009). En las Figs. 1, 2 y 3 se muestran interfaces del inicio del sistema, preguntas que tienen que ver con el estilo de aprendizaje y nivel de dificultad de los problemas que logran resolver los estudiantes.

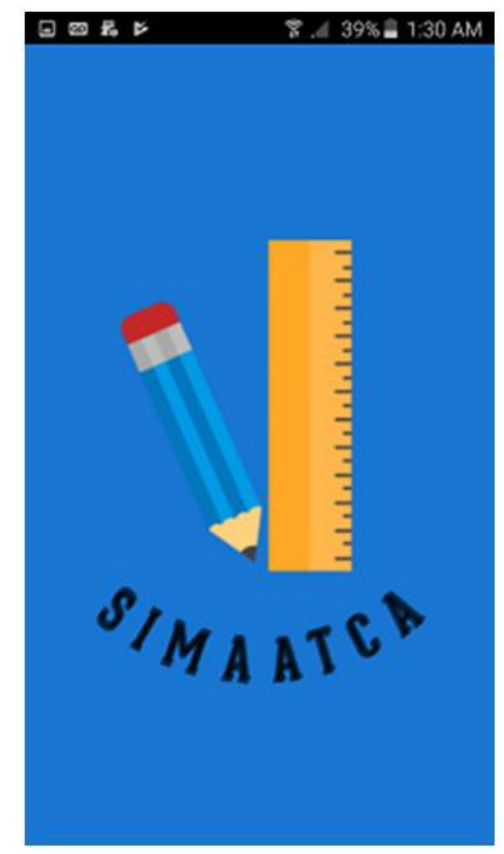

Fig. 1. Interfaz que muestra el inicio del Sistema SIMAATCA.

Fuente: Elaboración propia 


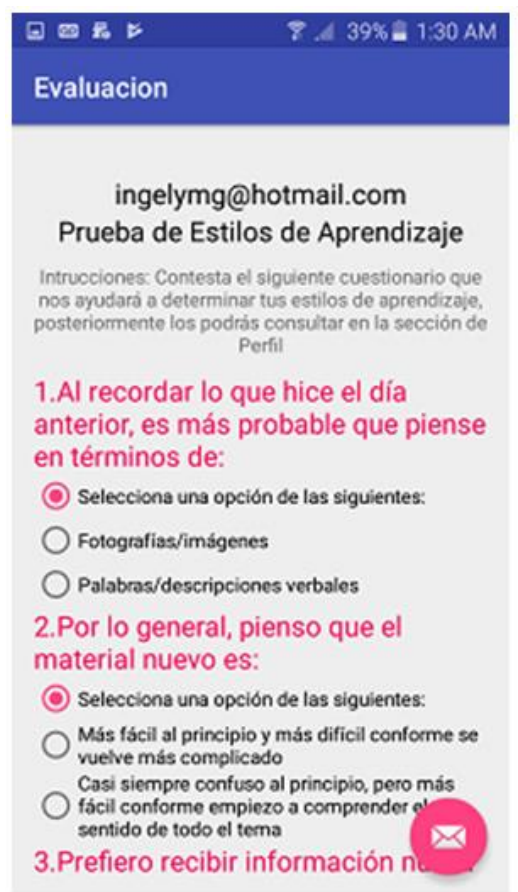

Fig. 2. Interfaz que muestra una pregunta del cuestionario, referente a estilos de aprendizaje.

Fuente: Elaboración propia.

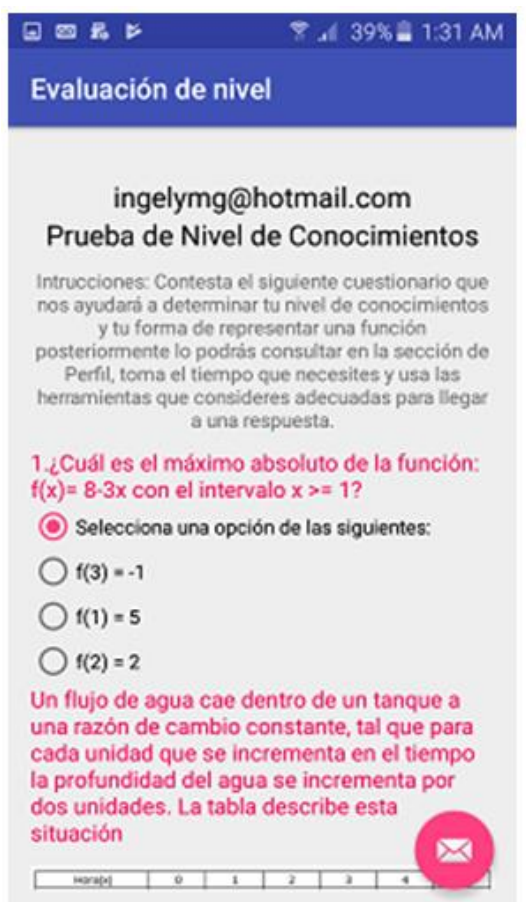

Fig. 3. Interfaz gráfica con preguntas para detectar el nivel de dificultad.

Fuente: Elaboración propia. 
Estudio sobre las dificultades que tienen estudiantes de nivel superior en la resolución de problemas de cálculo, y empleo de un Escenario de aprendizaje tecnopedagógico como apoyo en el trabajo del aula

\section{Aspectos teóricos a considerar en el trabajo del aula con el Escenario de aprendizaje Tecnopedagógico}

El trabajo realizado en el aula con el EATP consideró tomar en cuenta la visualización matemática, ya que ayuda al estudiante en la construcción de una estructura cognitiva sobre el control de la actividad matemática. Además, un alumno que no logra trabajar con las manipulaciones algebraicas, puede encontrar apoyo en la visualización y la aritmética para entender y controlar los resultados obtenidos en los procesos algebraicos.

De acuerdo con este enfoque, la experimentación y el análisis de los resultados se realizaron siguiendo un marco teórico sobre la visualización matemática (según Zimmermann \& Cuningham, 1991), sobre los procesos de control (según Saboya, 2010) que los estudiantes desarrollan para llevar a cabo una actividad matemática específica, y alrededor de la transición de la aritmética al álgebra (según Kaput, 2000, 2008; Kieran, 2007; y Artigue, 2011).

\section{Aspectos metodológicos}

\section{Sobre el modelo TPACK}

Con relación al Escenario de Aprendizaje Tecnopedagógico (EATP), éste se apoyó, para su construcción, en el Modelo TPACK (Technological Pedagogical Content Knowledge). Sobre este modelo se tiene lo siguiente: en la década de los 80, Shulman (1986), introdujo su modelo denominado Pedagogical Content Knowlegde, reducido a PCK, por sus siglas. Él señala que en la enseñanza no solo es requerido el dominio de la materia o de la disciplina con la que se va a trabajar, sino la forma, o las diferentes maneras que encuentra el profesor para representar el contenido que lo haga accesible a los estudiantes, es decir Shulman enfatiza la importancia que tiene la manera en que el tema es transformado para la enseñanza.

Los aspectos o ejemplos que Shulman considera como importantes para PCK son las analogías, ilustraciones, ejemplos, explicaciones y demostraciones, o, en otro palabras, las formas de representar y formular el tema para hacerlo más accesible y comprensible al estudiante.

Koehler y Mishra (2006), observaron que las tecnologías juegan un papel crítico en cada uno de estos aspectos. Desde dibujos en una pizarra, hasta interactivos o simulaciones multimedia o hipertextos basados en la web; por lo que las tecnologías han permitido una gama de representaciones, ejemplos, explicaciones y demostraciones que pueden ayudar a hacer los contenidos de una disciplina más accesible para el alumno. 
Por lo tanto, Koehler y Mishra (2006), consideran que el conocimiento de la tecnología se convierte en un aspecto importante del conocimiento general del docente. Lo que es interesante es que las discusiones actuales sobre el papel del conocimiento de la tecnología parecen compartir muchos de los mismos problemas que Shulman identificó en la década de 1980. Por ejemplo, antes del trabajo seminal de Shulman en $P C K$, el conocimiento del contenido y el conocimiento de la pedagogía fueron considerados separados e independientes el uno del otro. Del mismo modo, hoy, el conocimiento de la tecnología a menudo se considera separado del conocimiento de la pedagogía y contenido.

El conocimiento del contenido pedagógico y tecnológico (TPACK) implica entrelazar el qué enseñar, (contenido), con el cómo enseñar (mediante tecnología) y el para qué enseñarlo (pedagogía); es decir se requiere de que el profesor cuente con una formación en aspectos pedagógicos, pero no generales, sino aquellos que permita darse cuenta de las dificultades que un estudiante enfrenta al abordar una disciplina, además requiere contar con conocimiento de las nuevas tecnologías y su uso en cuestiones educativas.

Por lo tanto, el modelo de integración de la tecnología en la enseñanza y el aprendizaje propicia el desarrollo de un buen contenido mediante un entretejido reflexivo de las tres fuentes clave de conocimiento: tecnología, pedagogía y contenido. El núcleo de esto es que no hay una solución tecnológica única que aplica para cada maestro, cada curso o cada punto de vista de la enseñanza. Para que haya calidad en la enseñanza se requiere desarrollar una comprensión del complejo de relaciones entre tecnología, contenido y pedagogía, y el uso de esta comprensión para desarrollar estrategias apropiadas, específicas al contexto y representaciones.

\section{Estrategia para aplicar el modelo de TPACK}

Harris and Hofer (2009) señalan que antes de seleccionar los recursos tecnológicos como parte del proceso de enseñanza aprendizaje, se requiere tener claros los objetivos curriculares que se desean alcanzar en relación a la materia o disciplina con la que se va a trabajar, así como elegir las estrategias que permitirán un involucramiento del estudiante con el contenido a abordar (Harris And Hofer, 2009; Flores, 2013).

Para la implementación del modelo TPACK, Harris y Hofer (2005) proponen una serie de pasos a seguir, los cuales fueron tomados en consideración para el trabajo que se expone en este artículo: 
Estudio sobre las dificultades que tienen estudiantes de nivel superior en la resolución de problemas de cálculo, y empleo de un Escenario de aprendizaje tecnopedagógico como apoyo en el trabajo del aula

1. Determinar los objetivos de la disciplina con la que se va a trabajar.

2. Formular actividades.

3. Determinar estrategias de enseñanza y de aprendizaje.

4. Escoger recursos tecnológicos que permitan llevar a cabo las actividades formuladas con las estrategias seleccionadas.

Además Harris y Hofer (2009) proponen una taxonomía de las actividades de matemáticas que se pueden emplear como parte de la planificación de las tareas que un docente lleva a cabo. Esta taxonomía se fundamenta en los 7 géneros relacionados con las actividades propuestas por los estándares del National Council of Teachers of Mathematics (NCTM). Los 7 géneros considerados son:

1) Actividades para que el estudiante tenga su primer acercamiento con el tema.

2) Actividades para que el estudiante practique.

3) Actividades para que el estudiante interprete.

4) Actividades para producir.

5) Actividades para aplicar.

6) Actividades para evaluar.

\section{Método}

\section{Descripción del contexto}

El estudio se desarrolló en una de las Unidades Académicas del Instituto Politécnico Nacional (ESCOM IPN), con dos grupos de 33 estudiantes cada uno; estos grupos son constituidos por la institución de acuerdo al promedio que obtuvieron los estudiantes en su primer semestre. Los alumnos desde segundo semestre y hasta el octavo, eligen el horario en el que van a cursar las materias establecidas en la currícula, para ello a los estudiantes se les asigna un día y una hora en la que se pueden inscribir, esta asignación obedece al promedio que obtuvieron en primer semestre, de tal forma que el primer día de inscripciones se matriculan los alumnos con más alto promedio y así sucesivamente. Los dos grupos seleccionados correspondían al segundo semestre de la carrera de Ingeniería en Sistemas computacionales. Los estudiantes estaban inscritos en la asignatura de Cálculo Aplicado y como antecedente, habían cursado la materia de Cálculo en su 
primer semestre. Ambos grupos del estudio estaban formados por estudiantes cuyo promedio en el primer semestre oscilaba entre 4 y 6 (en una escala de 0 a 10), y cerca de la mitad había reprobado la materia de Cálculo en el primer semestre. Se decidió que fuera así para poder revisar cuáles eran sus errores y hacer el seguimiento de los estudiantes, para apoyarlos en la comprensión de conceptos fundamentales, en la resolución de problemas y que lograran enfrentar con éxito los exámenes a Título de Suficiencia. Un grupo se consideró como el grupo de control GC y el otro como el grupo experimental GE. La selección fue aleatoriamente, ya que los estudiantes de ambos grupos se encontraban en situaciones similares académicamente. En el grupo de control, $47 \%$ de los alumnos había reprobado la asignatura de Cálculo y del 53\% restante, la mitad había acreditado con la mínima calificación que es 6. La situación del grupo Experimental era similar, en éste, el 43\% había reprobado la materia de Cálculo y del 57\% restante el 37\% había obtenido la calificación de 6. Los promedios de los dos grupos eran bajos. El promedio del GC era de 2.6 y el promedio del GE era de 2.7 .

\section{Diseño de la investigación}

Por las características del diseño del estudio, es decir, el trabajar con dos grupos ya constituidos, aplicar un pre-test y un post-test, se utilizó con un diseño cuasi experimental. Ya que los diseños que carecen de un control experimental absoluto de todas las variables relevantes debido a la falta de aleatorización ya sea en la selección aleatoria de los sujetos o en la asignación de los mismos a los grupos experimental y control, que siempre incluyen una pre prueba para comparar la equivalencia entre los grupos, y que posiblemente poseen dos grupos (el experimental y el control), son conocidos con el nombre de cuasi experimentos. El método cuasi experimental es particularmente útil para estudiar problemas en los cuales no se puede tener control absoluto de las situaciones, pero se pretende tener el mayor control posible, aun cuando se estén usando grupos ya formados. Por ello, una característica de los cuasi experimentos es el incluir "grupos intactos", es decir, grupos ya constituidos. Algunas de las técnicas mediante las cuales se puede recopilar información en un estudio cuasi experimental son las pruebas estandarizadas, las entrevistas, las observaciones, etc. Se recomienda emplear en la medida de lo posible la pre prueba, es decir, una medición previa a la aplicación del tratamiento, a fin de analizar la equivalencia entre los grupos., 
Estudio sobre las dificultades que tienen estudiantes de nivel superior en la resolución de problemas de cálculo, y empleo de un Escenario de aprendizaje tecnopedagógico como apoyo en el trabajo del aula

En la búsqueda de la información proporcionada por los estudiantes, se recurrió al diseño y aplicación de dos cuestionarios, los cuales fueron aplicados de forma previa y posterior al trabajo en el aula con el Modelo Tecnopedagógico al que se le denominó Escenario de Aprendizaje Tecnopedagógico (EATP) construido para el grupo experimental (GE), y sin el empleo de éste para el grupo de control (GC). El primer cuestionario tuvo la finalidad de hacer un diagnóstico y el segundo fue de evaluación. Ambos cuestionarios se aplicaron de forma escrita a los dos grupos, y fueron empleados para medir el rendimiento académico de los dos grupos mediante la comparación de ambas calificaciones.

\section{Preguntas de los cuestionarios}

El primer cuestionario tuvo la función de realizar un diagnóstico de los grupos y el segundo permitió evaluar el avance en cada tema de la Unidad 1. En Tabla 1 aparecen las preguntas del cuestionario diagnóstico y en el cuadro 2 se encuentran las preguntas del cuestionario final. Sobre la fiabilidad y validez de los ítems o problemas del cuestionario, estos fueron extraídos de dos libros de texto que la Academia de Ciencias Básicas de la Unidad Académica donde se realizó el estudio, emplea, y se encuentran en el Plan y Programa de Estudios de la materia de Cálculo Aplicado (Plan de estudios de Cálculo Aplicado, ESCOM-PN, 2009, p. 8). Estos problemas son usados por los profesores en los exámenes que aplican a sus grupos, por ello es que se son considerados para los cuestionarios aplicados a los dos grupos del estudio.

Tabla 1. Problemas del cuestionario diagnóstico.

\begin{tabular}{|l|l|}
\hline \multicolumn{1}{|c|}{ Tema } & \multicolumn{1}{|c|}{ Problema } \\
\hline Razones de cambio relacionadas & $\begin{array}{l}\text { 1.- Un depósito para agua tiene la forma de un cono circular invertido, el radio } \\
\text { de la base es de } 2 \mathrm{~m} \text {, y la altura es de } 4 \mathrm{~m} . \text { Si el agua se bombea hacia el } \\
\text { depósito a una razón de } 2 \mathrm{~m}^{3} / \mathrm{min} \text {, determine la rapidez a la cual el nivel del } \\
\text { agua sube cuando el agua tiene } 3 \mathrm{~m} \text {. de profundidad. }\end{array}$ \\
\hline Diferencial de una función & $\begin{array}{l}\text { 2.- La dosis } \mathrm{D} \text { en miligramos de difenhidramina para un perro de masa } \\
\text { corporal } \mathrm{w} \mathrm{kg} \text { es de } D=4.7 \mathrm{w}^{\frac{2}{3}} \text {. Estime el máximo error admisible en w, para } \\
\text { un Cocker Spaniel de masa w=10kg, si el porcentaje de error es la dosis D no } \\
\text { puede superar el 3\%. }\end{array}$ \\
\hline Optimización & $\begin{array}{l}\text { 3.- Un veterinario cuenta con } 30 \mathrm{~m} \text { de tela de alambre y quiere construir } 6 \\
\text { jaulas para perros levantando primero una cerca alrededor de una región } \\
\text { rectangular, y dividiendo luego la región en 6 rectángulos iguales mediante 5 } \\
\text { rejas paralelas a uno de los lados. ¿Cuáles son las dimensiones de la zona } \\
\text { rectangular para las que el área total es máxima? }\end{array}$ \\
\hline $\begin{array}{l}\text { Análisis de una función } \\
\text { obteniendo puntos críticos, }\end{array}$ & $\begin{array}{l}\text { 4.- Determinar los puntos críticos de } f \text {, los puntos de inflexión de } f \text {, los } \\
\text { máximos y mínimos locales de } f \text {, determinar los intervalos en los que } f \text { es }\end{array}$ \\
\hline
\end{tabular}

№ 23, Vol. 11 (2), 2019. ISSN 2007 - 0705, pp.: 258 - 295 


\begin{tabular}{|l|l|}
\hline $\begin{array}{l}\text { intervalos de crecimiento y } \\
\text { concavidad }\end{array}$ & $\begin{array}{l}\text { creciente o decreciente, determinar los intervalos de concavidad de } f \text { y trazar } \\
\text { su gráfica. } \\
\mathrm{f}(\mathrm{x})=200+8 \mathrm{x}^{3}+\mathrm{x}^{4}\end{array}$ \\
\hline Optimización & $\begin{array}{l}\text { 5.- Encuentre las dimensiones de un rectángulo con un perímetro de 100 } \\
\text { metros, cuya área sea tan grande como sea posible. }\end{array}$ \\
\hline
\end{tabular}

Fuente: Stewart (2012), Larson \& Edwards (2010).

Tabla 2. Problemas del cuestionario final.

\begin{tabular}{|c|c|}
\hline Tema & Problema \\
\hline $\begin{array}{l}\text { Razones de cambio } \\
\text { relacionadas }\end{array}$ & $\begin{array}{l}\text { 1.- El automóvil A se dirije hacia el oeste a } 50 \frac{\text { millas }}{h} \text { y el vehículo B viaja hacia } \\
\text { el norte a } 60 \frac{\text { millas }}{h} \text {. Ambos se dirigen hacia la intersección de los dos caminos. } \\
\text { ¿Con qué rapidez se aproximan los vehículo entre sí cuando el autmóvil A está } \\
\text { a } 0.3 \text { millas y el vehículo B está a } 0.4 \text { millas de la intersección? }\end{array}$ \\
\hline Diferencial de una función & $\begin{array}{l}\text { 2.- Calcule el error relativo cometido en el cálculo del volumen de una esfera de } \\
12.51 \mathrm{~mm} \text { de diámetro, con un posible error de } 0.01 \mathrm{~mm} \text {, medido con un } \\
\text { instrumento que aprecia milésimas de centímetro. }\end{array}$ \\
\hline Optimización & $\begin{array}{l}\text { 3.- Los márgenes superior e inferior de un póster miden } 6 \mathrm{~cm} \text { y los márgenes } \\
\text { laterales miden } 4 \mathrm{~cm} \text {. Si el área del póster se fija en } 384 \mathrm{~cm}^{2} \text {, determine las } \\
\text { dimensiones del póster cuya área sea la mínima. }\end{array}$ \\
\hline $\begin{array}{l}\text { Análisis de una función } \\
\text { obteniendo puntos críticos, } \\
\text { intervalos de crecimiento y } \\
\text { concavidad }\end{array}$ & $\begin{array}{l}\text { 4.- Determinar los puntos críticos de } f \text {, los puntos de inflexión de } f \text {, los máximos } \\
\text { y mínimos locales de } f \text {, determinar los intervalos en los que } f \text { es creciente o } \\
\text { decreciente, determinar los intervalos de concavidad de } f \text { y trazar su gráfica. } \\
\qquad f(x)=2+3 x-x^{3}\end{array}$ \\
\hline Optimización & $\begin{array}{l}\text { 5.- Un terreno tiene la forma de un rectángulo con dos semicírculos en los } \\
\text { extremos. Si el perímetro del terreno es de } 50 \mathrm{~m} \text {, encontrar las dimensiones del } \\
\text { terreno para que tenga el área máxima. }\end{array}$ \\
\hline
\end{tabular}

Fuente: Stewart (2012), Larson \& Edwards (2010).

\section{Propuesta de trabajo}

La duración del trabajo en ambos grupos fue de un mes, y se trabajaron los contenidos correspondientes a la Unidad 1 de la asignatura de Cálculo Aplicado de una Unidad Académica del IPN (ESCOM-IPN). Durante este mes se tuvieron 3 sesiones por semana, de 1.5 horas cada 
sesión; en total fueron 11 sesiones. El trabajo fue realizado en el semestre de agosto a diciembre de 2018. Con el GE se trabajó con el Escenario de Aprendizaje Tecnopedagógico (EATP), empleando para ello el Sistema SIMAATCA el cual asigna recursos multimedia a los estudiantes, de acuerdo a su estilo de aprendizaje y a su nivel de dificultad, además le permite al estudiante resolver exámenes y revisar su avance registrando toda actividad realizada por él. Para la recolección de los datos, así como la construcción de gráficas se empleó Microsoft Excel. También se llevó a cabo un seguimiento del desarrollo de las competencias digitales de los estudiantes, debido a la integración de las diferentes herramientas tecnológicas dentro de un entorno de aprendizaje.

\section{Contenido matemático abordado}

Con ambos grupos se trabajó la Primera Unidad temática de la asignatura de Cálculo Aplicado de la ESCOM. Pero la forma de trabajo fue diferente en cada grupo.

La Unidad 1 se titula Aplicaciones de la derivada y los temas que se trabajaron fueron:

- Razones de cambio relacionadas.

- Diferencial de una función: Aplicaciones de la diferencial y determinación de errores.

- Aplicaciones de Máximos y mínimos: Criterio de la primera y segunda derivada.

- Problemas de optimización.

\section{Forma de trabajo con el grupo de control (GC)}

Con el grupo de Control se trabajó sin emplear el sistema SIMAATCA, solo se usó la calculadora y, la secuencia de enseñanza no se apoyó en el modelo TPACK. El maestro encargado del grupo permitió al investigador entrar a sus clases durante el mes que duró el trabajo realizado con la Unidad 1 del programa de estudio de la asignatura de Cálculo Aplicado.

En la tabla se describen las estrategias empleadas por el profesor, así como las actividades trabajadas con uno de los temas de la primera unidad y que es el de optimización. 
Tabla 3. Actividades y recursos empleados con el GC.

\begin{tabular}{|l|l|l|}
\hline \multicolumn{1}{|c|}{ Actividad } & Estrategia de enseñanza & \multicolumn{1}{c|}{ Recurso } \\
\hline $\begin{array}{l}\text { Definición de problemas de } \\
\text { optimización por parte del } \\
\text { profesor }\end{array}$ & $\begin{array}{l}\text { Expositiva } \\
\text { Memorística }\end{array}$ & Pizarrón y libro de texto \\
\hline $\begin{array}{l}\text { El maestro emplea los criterios de } \\
\text { la primera y segunda derivada al } \\
\text { resolver un problema de } \\
\text { optimización como ejemplo }\end{array}$ & Resolución de problemas & Pizarrón y calculadora \\
\hline $\begin{array}{l}\text { El maestro resuelve un segundo } \\
\text { ejemplo }\end{array}$ & Resolución de problemas & $\begin{array}{l}\text { Pizarrón, libro de texto y } \\
\text { calculadora }\end{array}$ \\
\hline $\begin{array}{l}\text { El maestro deja de tarea ejercicios } \\
\text { para resolver }\end{array}$ & Pizarrón y libro de texto \\
\hline
\end{tabular}

Fuente: Elaborada por el autor.

\section{Construcción del Escenario de Aprendizaje Tecnopedagógico (EATP)}

El Escenario de Aprendizaje Tecnopedagógico estuvo constituido por 3 componentes: uno correspondiente al contenido de matemáticas, en el presente artículo se muestra el tema de optimización, el segundo componente se refiere al aspecto pedagógico, de tal forma que para abordar el tema se empleó el enfoque de resolución de problemas, así como el uso de diferentes estrategias propuestas por la National Council Teaching of Mathematics (NCTM) y, en relación al tercer componente, que es el tecnológico, se usó el Sistema SIMAATCA que puede ser empleado en una computadora de escritorio, o en una computadora portátil, así como en una tableta o en un celular. En este caso se trabajó con dispositivos móviles, las tabletas y los celulares de los estudiantes (Drijvers, Kieran, Mariotti, Ainley, Andresen, Chan, And Meagher, 2010).

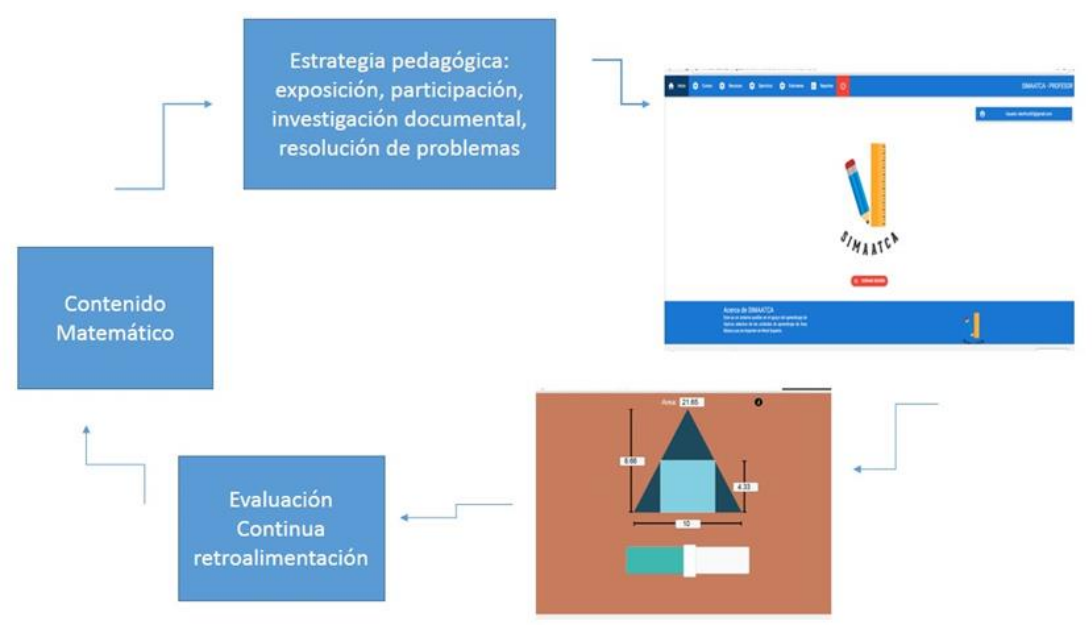

Fig. 4. Escenario de Aprendizaje Tecnopedagógico.

Fuente: Elaborado por el autor. 
Estudio sobre las dificultades que tienen estudiantes de nivel superior en la resolución de problemas de cálculo, y empleo de un Escenario de aprendizaje tecnopedagógico como apoyo en el trabajo del aula

\section{Forma de trabajo con el grupo experimental (GE)}

Con el Grupo experimental GE se trabajó empleando las etapas establecidas por Harris y Hofer (2005) para el modelo TPCAK, mencionadas en el apartado previo del presente documento, también se determinaron 2 de los 7 géneros señalados por la NCTM para el trabajo de las actividades.

Primer paso: Determinar los objetivos de aprendizaje de la asignatura con la que se va a trabajar, (Plan de estudios de Cálculo Aplicado, ESCOM-PN, 2009, p. 3).

El objetivo general de esta asignatura es:

Desarrollar habilidades lógico-matemáticas y destrezas, que le permitan al estudiante, mediante el razonamiento, el análisis y la reflexión, manejar las herramientas del Cálculo, para usarlas en la resolución de problemas que lleguen a presentarse en el ejercicio y desarrollo de su profesión.

El objetivo particular de la primera unidad temática consiste en "Aplicar los criterios de la primera y segunda derivada para resolver problemas de optimización", (ESCOM-IPN, 2009, p.3).

Segundo paso: Selección y secuenciación de las actividades. Para este segundo paso, primero se hizo una planeación de los temas que se trabajarían en cada sesión, así como las actividades que serían empleadas para cada tema. Como se muestra en el cuadro 4.

Tercer paso: Selección de las estrategias para trabajar con las actividades. En este paso se seleccionaron diferentes estrategias que se emplearían para trabajar cada actividad.

De entre las estrategias empleadas se encuentran:

- Realizar una demostración empleando una simulación de la construcción de algún objeto o de la ocurrencia de un fenómeno, que tenga que ver con el contexto de la actividad propuesta.

- Leer un texto, referido al contexto del problema.

- Discutir en equipo el planteamiento y la propuesta de solución.

- Reconocer un patrón al trabajar con aritmética y llegar a una generalización, que sería la función o el modelo matemático del fenómeno o situación del problema que se está 
trabajando.

- Hacer cálculos en el proceso de solución del problema.

- Resolver ejercicios relacionados al tema.

- Resolver un enigma o reto, lo cual se refiere a que, en equipo o de forma individual, resolverían otro problema con un grado de dificultad más elevado.

Algunas de las estrategias empleadas se muestran en la Tabla 4.

Tabla 4. Organización de los temas y las actividades correspondientes.

\begin{tabular}{|c|c|c|}
\hline Sesión & Tema & Actividad/ estrategia \\
\hline $\begin{array}{l}\text { Primera } \\
\text { (lunes } 6 \text { de } \\
\text { agosto de } \\
2018 \text { ) }\end{array}$ & $\begin{array}{l}\text { Cuestionario } \\
\text { diagnóstico }\end{array}$ & Aplicación del cuestionario. \\
\hline $\begin{array}{l}\text { Segunda } \\
\text { (miércoles } 8 \\
\text { de agosto de } \\
\text { 2018) }\end{array}$ & $\begin{array}{l}\text { Razones de } \\
\text { cambio } \\
\text { relacionadas }\end{array}$ & $\begin{array}{l}\text { Resolución de problemas, primero empleando la estrategia de ensayo y error al } \\
\text { dar valores para calcular lo que se pretendía, de ahí se llega a una generalización } \\
\text { y se emplean fórmulas. Se observan animaciones que simulan los fenómenos a } \\
\text { los que hacen referencia los problemas. }\end{array}$ \\
\hline & & $\begin{array}{c}\text { Esto es, se pide calcular la derivada } \frac{\mathrm{dz}}{\mathrm{dt}} \text { cuando el } \\
\text { tiempo t transcurrido es de } 2 \mathrm{~h} \text {. La posición de los } \\
\text { barcos después de haber iniciado su } \\
\text { desplazamiento es: }\end{array}$ \\
\hline & & \\
\hline $\begin{array}{l}\text { Tercera } \\
\text { (jueves } 9 \text { de } \\
\text { agosto de } \\
\text { 2018) }\end{array}$ & $\begin{array}{l}\text { Razones de } \\
\text { cambio } \\
\text { relacionadas, } \\
\text { ejemplos y } \\
\text { solución de } \\
\text { ejercicios }\end{array}$ & Se resuelven problemas empleando diferentes técnicas. \\
\hline
\end{tabular}


Estudio sobre las dificultades que tienen estudiantes de nivel superior en la resolución de problemas de cálculo, y empleo de un Escenario de aprendizaje tecnopedagógico como apoyo en el trabajo del aula

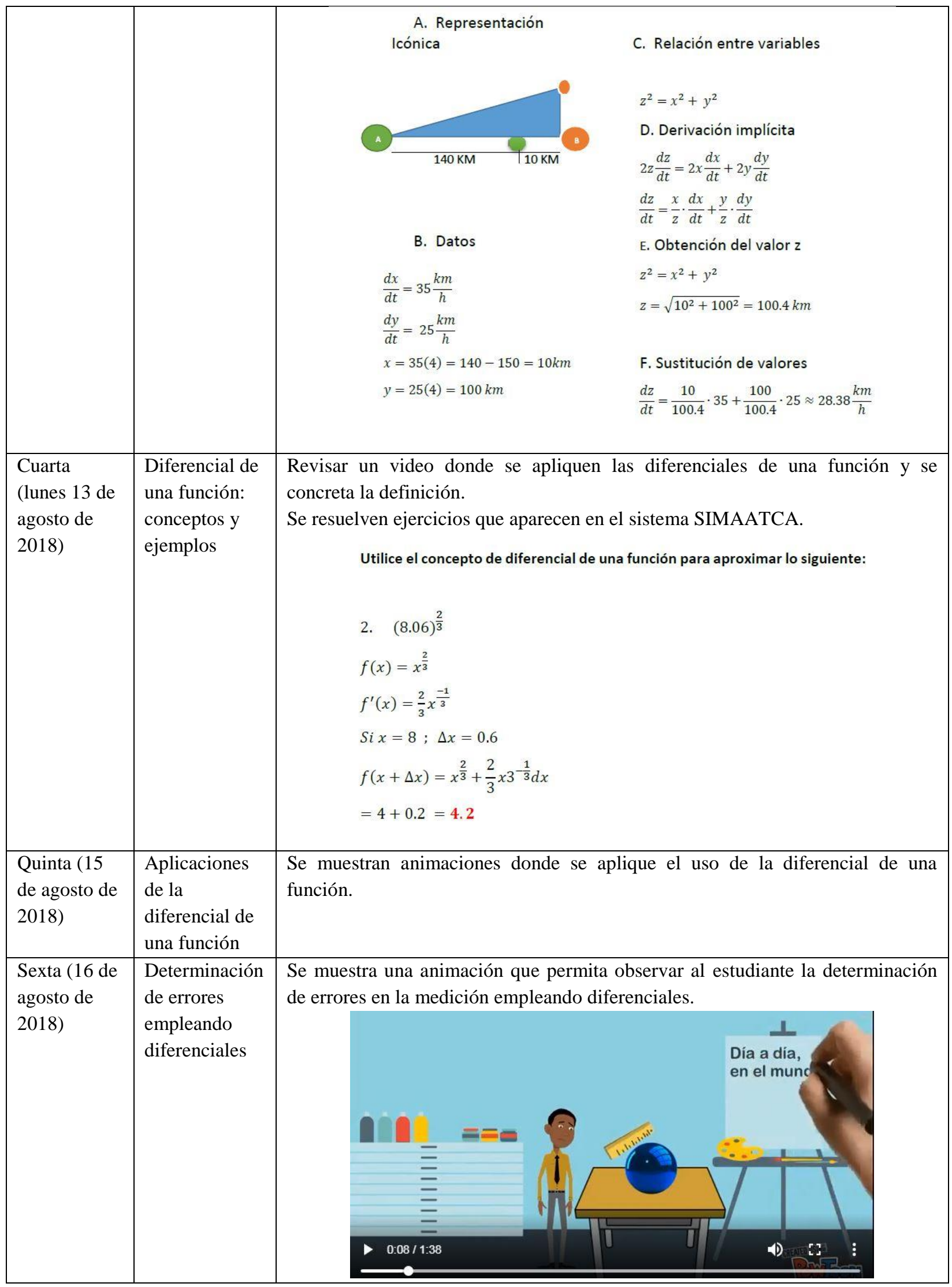




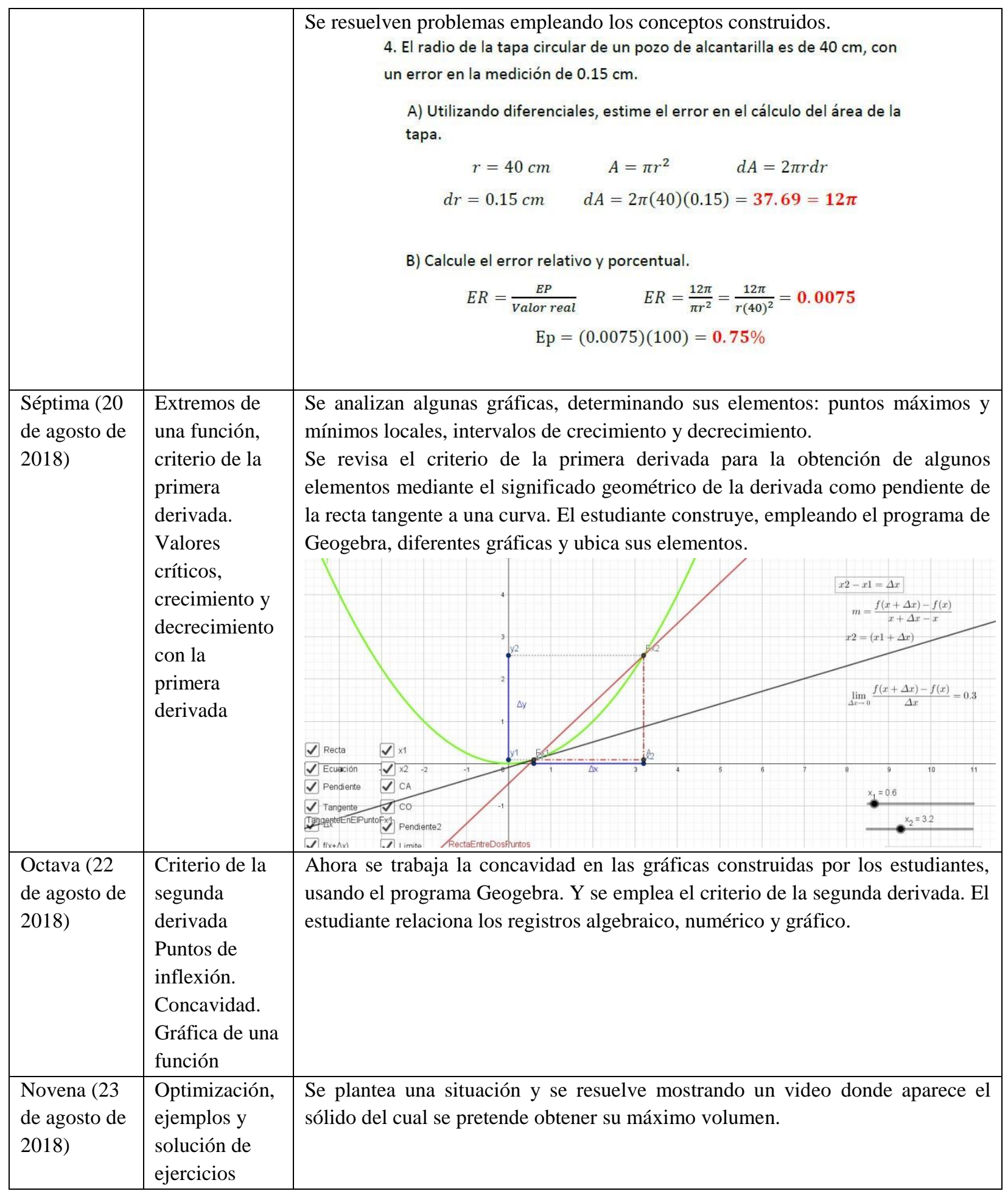


Estudio sobre las dificultades que tienen estudiantes de nivel superior en la resolución de problemas de cálculo, y empleo de un Escenario de aprendizaje tecnopedagógico como apoyo en el trabajo del aula

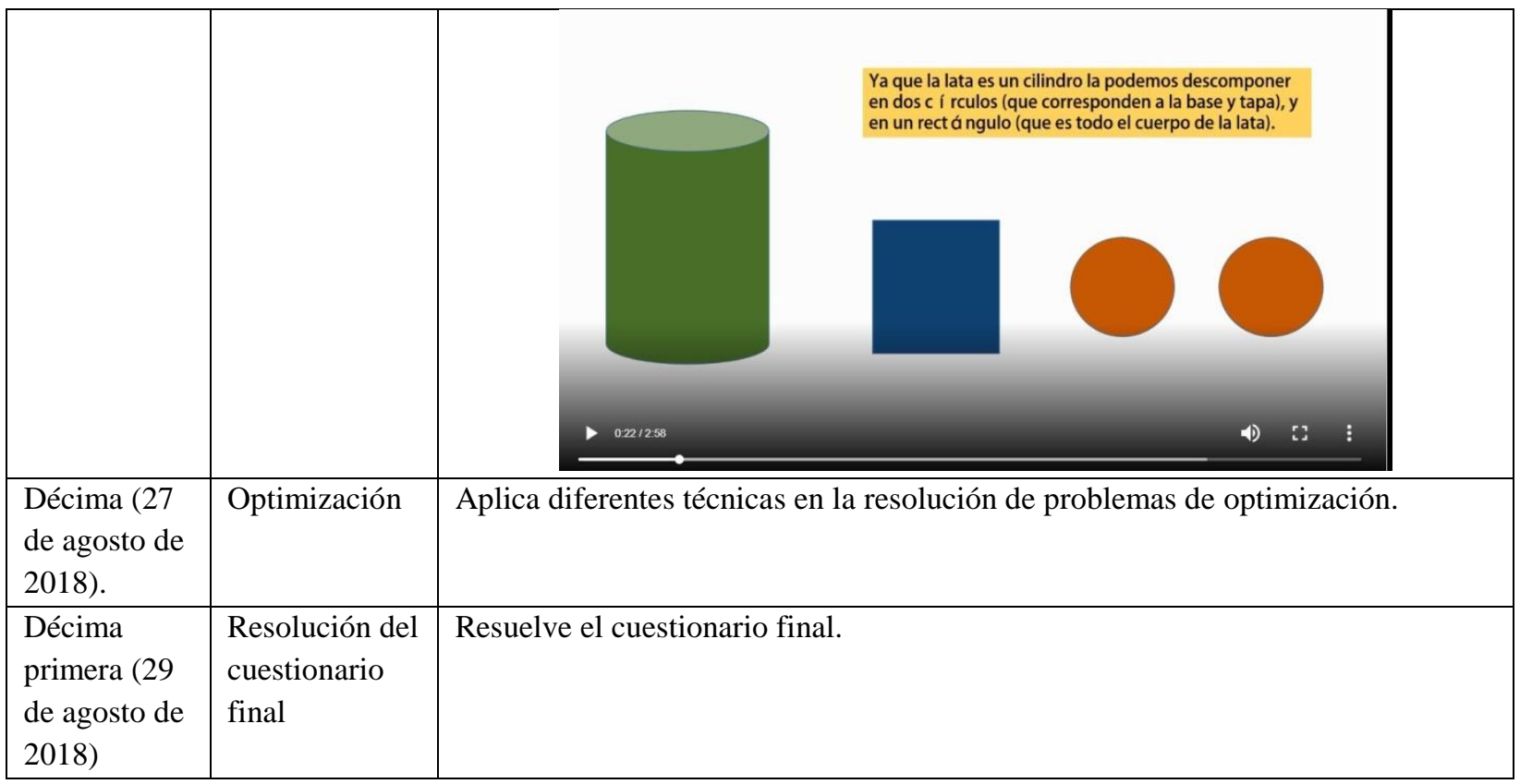

Fuente: Elaborada por el autor.

Cuarto paso: Selección de las tecnologías más adecuadas para el desarrollo de las actividades propuestas. Explicar las tecnologías usadas.

En la Tabla 5 se muestra un ejemplo, que es con el tema de Optimización, en el que se describe una actividad, las estrategias empleadas y los recursos tecnológicos utilizados con el GE.

Tabla 5. Actividades, estrategias y tecnología usadas en el caso del tema de optimización correspondiente a la unidad 1.

\begin{tabular}{|c|c|c|}
\hline Estrategia & Actividad & Tecnologías empleadas \\
\hline $\begin{array}{l}\text { Presenciar una } \\
\text { demostración }\end{array}$ & $\begin{array}{l}\text { El docente mostró a través de una animación del sistema } \\
\text { SIMAATCA, la optimización del volumen de una caja formada } \\
\text { por una hoja de cartón que tenía un área determinada. }\end{array}$ & $\begin{array}{l}\text { Presentación mediante } \\
\text { diapositivas. } \\
\text { Uso del Sistema } \\
\text { SIMAATCA para revisar la } \\
\text { animación del problema de } \\
\text { la construcción de la caja. }\end{array}$ \\
\hline Leer textos & $\begin{array}{l}\text { Los estudiantes Descargaron en su celular los apuntes que se } \\
\text { encuentran en SIMAATCA. Después, extrajeron información dell } \\
\text { tema de optimización. }\end{array}$ & $\begin{array}{l}\text { Libro electrónico contenido } \\
\text { en SIMAATCA }\end{array}$ \\
\hline
\end{tabular}




\begin{tabular}{|c|c|c|c|c|c|}
\hline Discutir & \multicolumn{4}{|c|}{$\begin{array}{l}\text { Los estudiantes discutieron en equipo el concepto de } \\
\text { optimización. }\end{array}$} & Tablet o cuaderno de notas. \\
\hline \multirow{5}{*}{$\begin{array}{l}\text { Reconocer un } \\
\text { patrón }\end{array}$} & \multicolumn{4}{|c|}{$\begin{array}{l}\text { Los estudiantes descargaron en su celular la animación de la } \\
\text { caja, junto con la tabla, interactuaron con ella al dar valores y } \\
\text { observar los volúmenes obtenidos. Observaron el patrón que se } \\
\text { repetía en la tabla, con los casos particulares que obtuvieron. }\end{array}$} & \multirow{5}{*}{$\begin{array}{l}\text { Programa interactivo para } \\
\text { introducir valores, llenar una } \\
\text { tabla y construir una gráfica } \\
\text { que se encuentra en } \\
\text { SIMAATCA }\end{array}$} \\
\hline & \begin{tabular}{||c|c} 
Altura \\
$(\mathbf{c m})$
\end{tabular} & $\begin{array}{c}\text { Largo } \\
(\mathrm{cm})\end{array}$ & $\begin{array}{l}\text { Ancho } \\
\text { (cm) }\end{array}$ & $\begin{array}{c}\text { Volumen } \\
\left(\mathrm{cm}^{3}\right)\end{array}$ & \\
\hline & 1 & $30-2(1)=28$ & $25-2(1)=23$ & $1(28)(25)$ & \\
\hline & 2 & $30-2(2)=26$ & $25-2(2)=21$ & $2(26(21)$ & \\
\hline & $\mathrm{X}$ & $30-2 x$ & $25-2 \mathrm{x}$ & $\begin{array}{l}x(30 \\
-2 x)(25 \\
-2 x)\end{array}$ & \\
\hline $\begin{array}{l}\text { Comprender o } \\
\text { definir un } \\
\text { problema }\end{array}$ & $\begin{array}{l}\text { Los estuc } \\
\text { los distin }\end{array}$ & $\begin{array}{l}\text { tes definiero } \\
\text { volúmenes ol }\end{array}$ & $\begin{array}{l}\text { a expresión } \\
\text { iidos proban }\end{array}$ & $\begin{array}{l}\text { ítica que generaliza } \\
\text { arios valores. }\end{array}$ & Uso del programa Geogebra \\
\hline
\end{tabular}

Fuente: Contenidos producidos en la investigación del autor.

Tabla 6. Descripción del uso de algunas estrategas con el apoyo de la tecnología.

\begin{tabular}{|c|c|c|}
\hline Estrategia & Breve descripción & Posibles tecnologías \\
\hline Hacer cálculos & $\begin{array}{l}\text { Los estudiantes emplearon la calculadora para } \\
\text { obtener los distintos volúmenes de la caja y } \\
\text { después usaron el criterio de la primera derivada } \\
\text { para determinar los valores que permiten } \\
\text { obtener el máximo volumen de la caja. }\end{array}$ & $\begin{array}{l}\text { Calculadoras científicas y } \\
\text { graficadoras, hoja de cálculo, } \\
\text { Programa Mathematica }\end{array}$ \\
\hline Hacer ejercicios y prácticas & $\begin{array}{l}\text { Los estudiantes resolvieron otro problema de } \\
\text { optimización practicando distintas estrategias } \\
\text { como el proponer valores numéricos. }\end{array}$ & $\begin{array}{l}\text { Sistema SIMAATCA. } \\
\text { Libros digitales. }\end{array}$ \\
\hline Resolver un enigma & $\begin{array}{l}\text { Los estudiantes implementan una estrategia o } \\
\text { técnica matemática dentro del contexto des } \\
\text { resolución de un enigma atractivo, que puede ser } \\
\text { facilitado o planteado a través de tecnología. }\end{array}$ & $\begin{array}{l}\text { Sitios web de juegos matemáticos } \\
\text { (por ejemplo, CoolMath) }\end{array}$ \\
\hline
\end{tabular}

Fuente: Contenidos producidos en la investigación del autor.

\section{Desenvolvimiento de los estudiantes del GE}

Durante el mes que duró el trabajo de la primera unidad empleando el Escenario de Aprendizaje Tecnopedagógico, que incluyó las estrategias, actividades y tecnología, señaladas en las Tablas 4, 5 y 6 , se encontró a los estudiantes motivados e interesados en lo que se presentaba y resolvía en clase, participativos, preguntaban dudas específicas en la resolución de los problemas, pasaban al 
Estudio sobre las dificultades que tienen estudiantes de nivel superior en la resolución de problemas de cálculo, y empleo de un Escenario de aprendizaje tecnopedagógico como apoyo en el trabajo del aula

pizarrón a resolver ejercicios y situaciones planteadas. Todos empleaban su celular con las actividades propuestas en el sistema SIMAATCA, hubo trabajo colaborativo. No se apreció desinterés por el trabajo en el aula ni distracciones. Los alumnos comentan que algunos problemas representaban retos, cuya solución les generaba un estado de satisfacción y los motivaba a continuar con el trabajo. Para poder medir el rendimiento de los estudiantes, se aplicó un cuestionario final y se compararon los resultados de las calificaciones obtenidas entre el primer cuestionario (el de diagnóstico) y el final, lo que se muestra en el siguiente apartado.

\section{$\underline{\text { Resultados }}$}

Los datos fueron tratados en Excel, empleando las fórmulas básicas de promedio y varianza, pero básicamente se utilizó una metodología cualitativa para analizar las respuestas dadas por los estudiantes a los cuestionarios aplicados, lo que permitió documentar y analizar los procesos seguidos por los estudiantes en la resolución de los problemas. (Hernández Sampieri, 2014). Moschkovich (2000) señala que los procesos empleados en la construcción de conceptos se dan a través de la práctica, lo que se tomó en cuenta al analizar como los alumnos empleaban los recursos multimedia del Escenario tecnopedagógico. Se emplearon aspectos cuantitativos para establecer la diferencia estadísticamente significativa, se analizaron los datos del Grupo control (GC) y del Grupo de estudio (GE), en una etapa inicial (I), que fue antes de trabajar con el EATP y al final del trabajo en el aula (F). El nombre de las columnas quedó como GC_I, GC_F, GE_I y GE_F.

Primero se analizó la gaussianidad de las muestras, y todas las columnas pasaron la prueba de normalidad de D' Agostino \& Pearson (ver Tabla 7). En seguida se aplicó el análisis ANOVA una vía y se encontraron diferencias entre los cuatro casos $(\mathrm{p}<0.5)$. Enseguida se analizó si había diferencias significativas bajo tres casos: entre las condiciones iniciales, entre la condición inicial y final de cada grupo y entre las condiciones finales de cada grupo. Empleando la prueba t-student, no pareada con corrección de Welch y considerando dos colas en todos los casos. Se comprobó que ambos grupos al inicio del estudio se encontraban en condiciones similares, con una $\mathrm{p}<0.05$ (ver Tabla 8.) Posteriormente al comparar las diferencias entre los resultados obtenidos de los cuestionarios, tanto el inicial como final de ambos grupos, los resultados entre las etapas son diferentes $(\mathrm{p}<0.0001)$ y al usar la prueba $\mathrm{F}$ para determinar diferencias en las varianzas de cada una, se obtuvo que ambos casos tienen una distribución 
similar con una $\mathrm{p}>0.05$. Se observa que para GE la diferencia entre el inicio y final fue de $4.121 \pm$ 0.4339 y para GC fue de $1.902 \pm 0.3843$. Finalmente, al comparar las diferencias entre los estados finales de los dos grupos se encontró una diferencia de $2.661 \pm 0.4162$ con p <0.0001.

Primero se analizó la gaussianidad de las muestras, y todas las columnas pasaron la prueba de normalidad de D'Agostino \& Pearson (ver Tabla 7). Enseguida se analizó si había diferencias significativas entre las 4 columnas por pares, lo que se muestra en la Tabla 8. Al realizar el análisis de las condiciones iniciales de los 2 grupos empleando la prueba t no pareada con corrección de Welch, se comprobó que ambos grupos al inicio del estudio se encontraban en condiciones similares, ya que se obtuvo una $\mathrm{p}<0.05$.

Tabla 7. Concentrado de información al aplicar la prueba de normalidad D'Agostino \& Pearson para medir la condición inicial del GC y del GE.

\begin{tabular}{|l|c|c|c|c|}
\hline \multicolumn{1}{|c|}{ Número de valores } & $\mathbf{3 5}$ & $\mathbf{3 2}$ & $\mathbf{3 3}$ & $\mathbf{3 3}$ \\
\hline Mínimo & 0.0 & 2.000 & 0.0 & 2.000 \\
\hline 25\% Percentil & 2.000 & 4.000 & 2.000 & 6.000 \\
\hline Mediana & 2.000 & 4.000 & 2.000 & 6.000 \\
\hline $75 \%$ Percentil & 4.000 & 6.000 & 4.000 & 8.000 \\
\hline Máximo & 6.000 & 6.000 & 6.000 & 10.00 \\
\hline & & & & \\
\hline $10 \%$ Percentil & 0.0 & 2.000 & 0.0 & 4.000 \\
\hline $90 \%$ Percentil & 4.800 & 6.000 & 4.000 & 10.00 \\
\hline & & & & \\
\hline Significancia & 2.286 & 4.188 & 2.727 & 6.848 \\
\hline Desviación estándar & 1.759 & 1.378 & 1.567 & 1.938 \\
\hline Std. Error de significancia & 0.2972 & 0.2436 & 0.2727 & 0.3374 \\
\hline Abajo del 95\% CI de significancia & & & & \\
\hline Arriba del 95\% CI de significancia & 1.682 & 3.691 & 2.172 & 6.161 \\
\hline & 2.890 & 4.684 & 3.283 & 7.536 \\
\hline Prueba de Normalidad D'Agostino \& Pearson & & & & \\
\hline K2 & & & & \\
\hline P valor & 1.883 & 1.386 & 0.05515 & 0.7290 \\
\hline Prueba de normalidad (alpha=0.05) & 0.3900 & 0.5000 & 0.9728 & 0.6946 \\
\hline P resumen del valor & $\mathbf{S i}$ & $\mathbf{S i}$ & $\mathbf{S i}$ & $\mathbf{S i}$ \\
\hline & $\mathrm{ns}$ & $\mathrm{ns}$ & $\mathrm{ns}$ & $\mathrm{ns}$ \\
\hline Coeficiente de variación & & & & \\
\hline & $76.94 \%$ & $32.91 \%$ & $57.45 \%$ & $28.30 \%$ \\
\hline Significancia geométrica & & & & \\
\hline & & 3.937 & & 6.526 \\
\hline Oblicuidad & 0.5338 & -0.1226 & 0.06863 & -0.3207 \\
\hline Kurtosis & -0.1652 & -0.7678 & -0.2593 & 0.002105 \\
\hline & & & & \\
\hline Suma & 80.00 & 134.0 & 90.00 & 226.0 \\
\hline
\end{tabular}


Estudio sobre las dificultades que tienen estudiantes de nivel superior en la resolución de problemas de cálculo, y empleo de un Escenario de aprendizaje tecnopedagógico como apoyo en el trabajo del aula

Tabla 8. Caso 1: condiciones iniciales de los 2 grupos.

\begin{tabular}{|c|c|c|c|}
\hline Análisis & Grupos & & \\
\hline Columna C & GE_I & & \\
\hline Vs. & Vs. & & \\
\hline Columna A & GC_I & & \\
\hline \multicolumn{4}{|l|}{ Prueba t no pareada con corrección de Welch } \\
\hline P valor & 0.2777 & & \\
\hline P resumen del valor & ns & & \\
\hline ¿Significativamente diferente? $(P<0.05)$ & No & & \\
\hline ¿Valor P de una o dos colas? & Dos-colas & & \\
\hline T corregida de Welch, df & $\mathrm{t}=1.095 \mathrm{df}=65.80$ & & \\
\hline \multicolumn{4}{|l|}{ ¿Qué tan grande es la diferencia? } \\
\hline Mean \pm SEM de la columna A & $2.286 \pm 0.2972, n=35$ & & \\
\hline Mean \pm SEM de la columna $C$ & $2.727 \pm 0.2727, n=33$ & & \\
\hline Diferencia entre los medios & $0.4416 \pm 0.4034$ & & \\
\hline $95 \%$ intervalo de confianza & -0.3639 to 1.247 & & \\
\hline R cuadrada & 0.01788 & & \\
\hline \multicolumn{4}{|l|}{ F prueba de comparación de varianzas } \\
\hline F,DFn, Dfd & $1.260,34,32$ & & \\
\hline P valor & 0.5136 & & \\
\hline P resumen del valor & $\mathrm{ns}$ & & \\
\hline ¿Significativamente diferente? $(\mathrm{P}<0.05)$ & No & & \\
\hline Modelo de comparación & SS & $\mathrm{DF}$ & La probabilidad es correcta \\
\hline Hipótesis Nula: Population means identical & 187.0 & 67 & $61.96 \%$ \\
\hline $\begin{array}{l}\text { Hipótesis Alternativa: Distinct population } \\
\text { means }\end{array}$ & 183.7 & 66 & $38.04 \%$ \\
\hline Razón de probabilidades & & & 1.629 \\
\hline Diferencia en AICc & & & -0.9753 \\
\hline
\end{tabular}

\section{Resultados del cuestionario diagnóstico}

Los resultados obtenidos del cuestionario diagnóstico tanto por el grupo de control (GC) como del grupo experimental (GE) fueron muy bajos como se muestra en las gráficas de las Figs. 5 y 6 , respectivamente. Los estudiantes presentaron dificultades en los planteamientos de la mayoría de los problemas. La tarea que tuvo mayor cantidad de respuestas correctas, en ambos grupos, fue la 
que se refería a la obtención de puntos máximos, mínimos, puntos de inflexión (problema 4 o P4 como aparece en la gráfica), aunque no todos los alumnos tuvieron correcto este problema debido a que no pudieron hacer la gráfica, lo que indica que no han logrado trabajar estableciendo la relación entre los diferentes registros de representación y no han llegado a construir el concepto de función completamente, sólo de forma parcial, como lo señala Duval (1998). La tarea que tuvo menos aciertos, también en ambos grupos, fue la correspondiente al uso de diferenciales para obtener las medidas solicitadas (problema 2 o P2 como se muestra en la gráfica). Los problemas de optimización (problemas 3 y 5 o P3 y P5), como el de razones de cambio relacionadas (problema 1 o P1) también fueron considerados como difíciles por los alumnos, al no poder establecer las relaciones con las variables dadas y con ello llegar a las respuestas solicitadas.

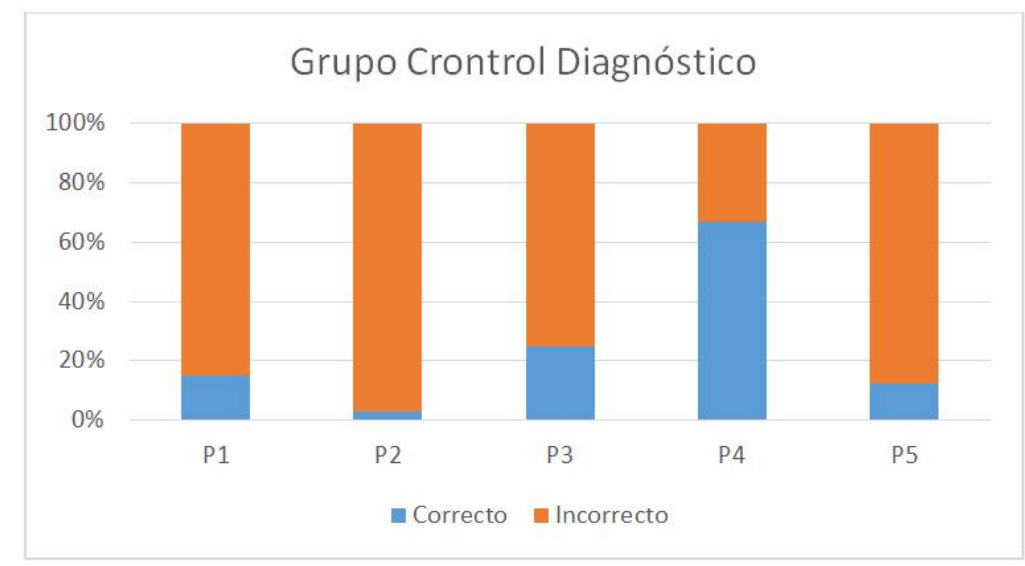

Fig. 5. Gráfica que representa los resultados del cuestionario diagnóstico que corresponden al grupo de control (GC).

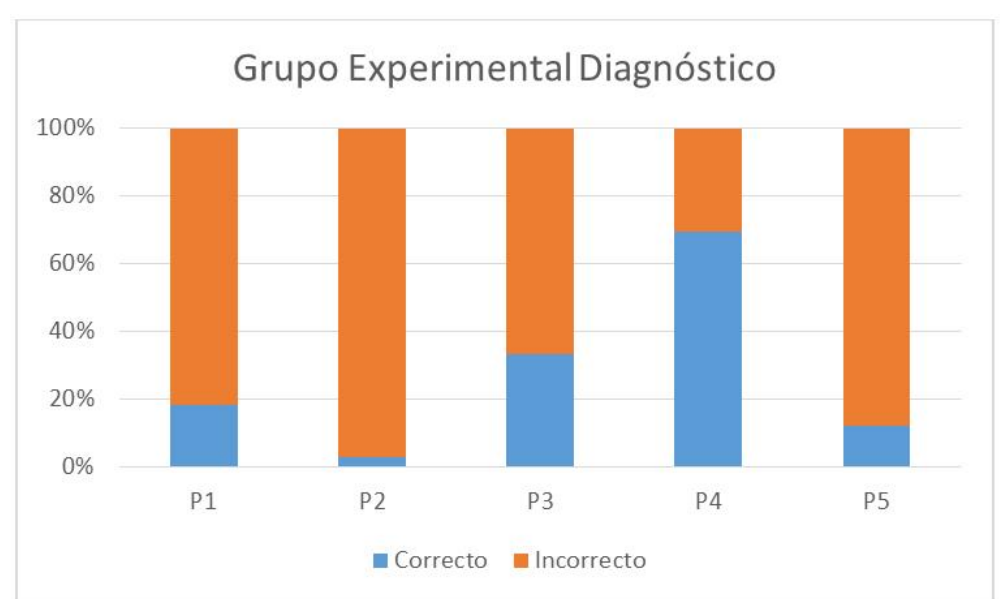

Fig. 6. Gráfica que representa los resultados del cuestionario diagnóstico que corresponden al grupo experimental (GE). 
Estudio sobre las dificultades que tienen estudiantes de nivel superior en la resolución de problemas de cálculo, y empleo de un Escenario de aprendizaje tecnopedagógico como apoyo en el trabajo del aula

\section{Análisis de los resultados obtenidos en el cuestionario diagnóstico por ambos grupos}

\section{1) Problemas de Razones de cambio relacionadas}

En relación a los problemas de razones de cambio relacionadas, en un inicio los estudiantes tanto del grupo de control como del grupo experimental, no identificaron los datos proporcionados como razones de cambio con respecto al tiempo y tampoco determinaron la ecuación que sufriría una modificación mediante el proceso de la obtención de la derivada, para al final obtener la razón de cambio que se requería encontrar. Los estudiantes no lograban relacionar las variables mediante una función auxiliar, como el establecimiento de triángulos semejantes, "para el problema del depósito que tiene forma de cono invertido". Esto muestra escaso trabajo en el campo de la geometría y su vinculación con el cálculo.

\section{2) Problemas de diferenciales}

Los estudiantes manifestaron desconocimiento del tema, pero ni siquiera hubo intentos por tener aproximaciones en la resolución; sólo 2 estudiantes, uno de cada grupo (el de control y el experimental), emplearon aritmética para aproximar uno de los errores en la medición solicitados. Para ello, primero encontraron el valor real del volumen del cubo y después el valor aproximado; al primero le restaron el segundo, encontrando tanto el error máximo como el mínimo en la medición del volumen del cubo. Esto implica que el $97 \%$ de los estudiantes siguen trabajando con procesos aritméticos sin haber llegado a la transición del conocimiento de los infinitesimales, mediante el cálculo, pero el grueso de los estudiantes no ha logrado construir un modelo matemático que les permita manipular tanto a las variables independientes como dependientes y obtener la diferencial de la función para llegar a responder a la situación planteada.

\section{3) Problemas de optimización}

En un inicio los estudiantes tuvieron dificultades para construir el modelo matemático que los ayudaría a encontrar la maximización o minimización de lo que el problema requería obtener. Los estudiantes han tenido durante varios años acercamiento con la aritmética, pero no están haciendo uso de ella para tener aproximaciones en lo que se requiere obtener, tampoco tienen habilidad en el uso de estrategias, como la estrategia de ensayo y error al probar distintos valores hasta llegar a una generalización. Pareciera que los estudiantes están muy mecanizados y se desesperan al no contar con una función a la que le van a aplicar el criterio de la primera o segunda derivada hasta 
llegar a la determinación del mínimo o máximo.

\section{Análisis de una función y su gráfica}

En general los estudiantes resolvieron la situación en la que se requería determinar los puntos críticos, los intervalos de crecimiento y concavidad. Se observa que cuando se da una función de forma analítica, los estudiantes hacen una manipulación algebraica siguiendo una serie de pasos establecidos, aquí la memoria jugó un importante papel, ya que siguieron pasos como derivar la función, igualar a cero, resolver la ecuación para $x$ y determinar qué valor de $x$ represente la abscisa de un punto que es máximo y cual la de un mínimo. Las dificultades encontradas tuvieron que ver con el hecho de no darle sentido al proceso de la determinación de máximos y mínimos, más bien se apoyaron en la memoria y no en el razonamiento.

\section{$\underline{\text { Resultados del cuestionario final }}$}

Después de haber trabajado con el grupo de Control (GC) los temas de la Unidad 1, de forma tradicional, en donde el maestro dictaba la clase empleando el pizarrón y un libro de texto, dando aspectos teóricos y ejemplos de problemas referente a la misma Unidad 1, y después de haber trabajado con el grupo Experimental (GE) a través del Escenario de Aprendizaje Tecnopedagógico, empleando una serie de problemas y el Sistema SIMAATCA, que contiene videos, presentaciones en PowerPoint, programas para hacer cálculos, animaciones y simulaciones, los estudiantes tanto del GC como del GE, resolvieron un cuestionario final, el cual, al igual que el cuestionario diagnóstico, constó de 5 problemas referentes a aplicaciones del Cálculo. En esta ocasión hubo una mejora en los resultados obtenidos por ambos grupos (el de control como el experimental), pero el GE sobresalió en la cantidad de respuestas correctas comparado con el GC.

En las Figs. 7 y 8 se muestran las gráficas de las respuestas correctas de cada grupo correspondientes tanto al cuestionario diagnóstico como al final. 


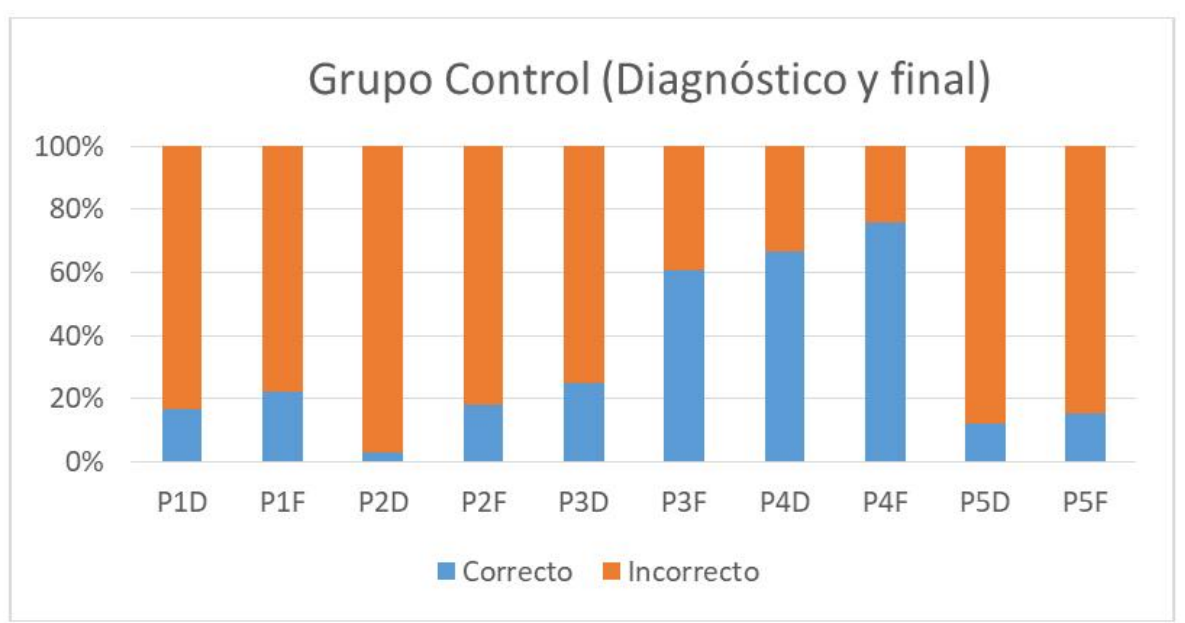

Fig. 7. Gráfica que representa los resultados tanto del cuestionario inicial como del cuestionario final correspondientes al grupo de control (GC).

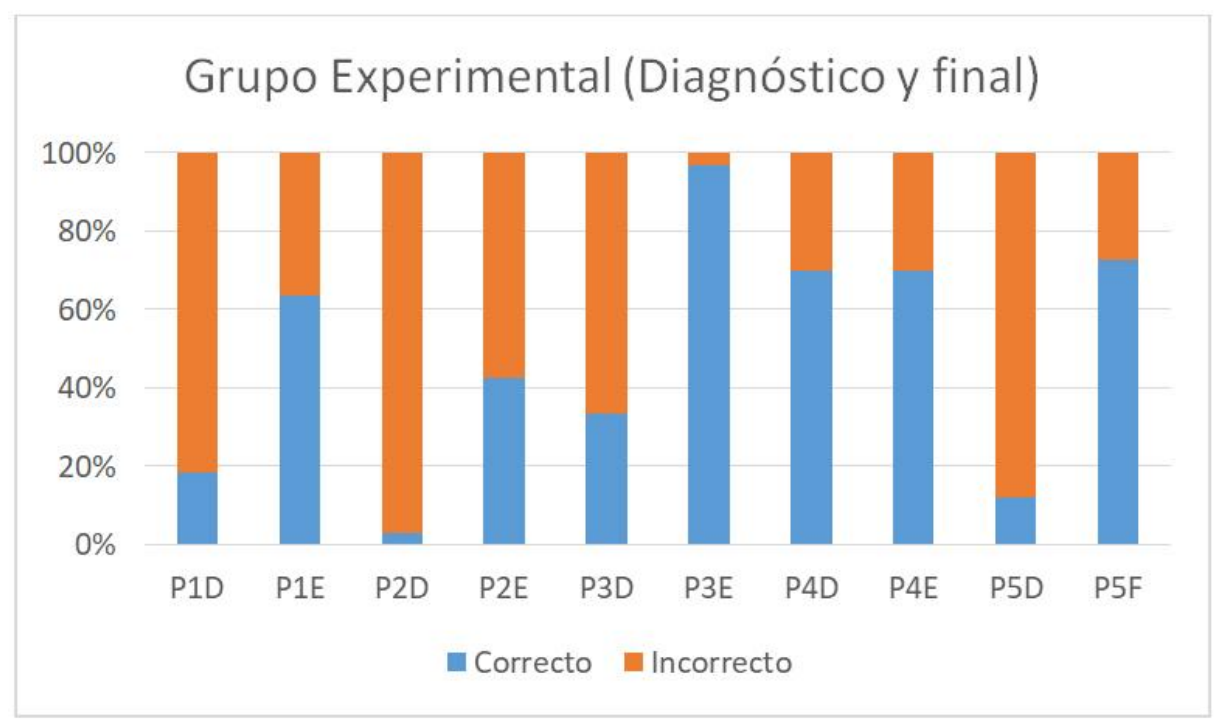

Fig. 8. Gráfica que representa los resultados del cuestionario final correspondiente al grupo experimental (GE).

En el grupo de control los problemas que fueron resueltos de forma correcta, por mayor cantidad de estudiantes, correspondieron a la obtención de puntos críticos (máximos, mínimos), intervalos donde la función es creciente y en donde es decreciente $(\mathrm{P} 4 \mathrm{~F}$, es decir, problema 3 del cuestionario final), así como el concerniente al tema de optimización (P3F), aunque en el segundo problema de optimización (P5F) no tuvieron mucho éxito, debido a la construcción geométrica y al uso de fórmulas de geometría que debían conocer y que olvidaron en el momento de la resolución del cuestionario. Los problemas en los que fallaron al rededor del $80 \%$ de los alumnos fueron del tema de razones de cambio relacionadas $(\mathrm{P} 1 \mathrm{~F})$ y del de diferenciales $(\mathrm{P} 2 \mathrm{~F})$. 
En la misma Fig. 7 se muestra la gráfica con el porcentaje de respuestas correctas como incorrectas de los dos cuestionarios aplicados, el de diagnóstico como el final (Fig. 7), para hacer una comparación entre ambos porcentajes, con lo cual se puede decir que hubo un avance y una mejora en el rendimiento de los alumnos del GC pues su promedio, aumentó de 2.4 a 3.7, en una escala de 0 a 10.

\section{Análisis de resultados del grupo de control}

Revisando cada uno de los problemas de los cuestionarios aplicados se muestran las dificultades encontradas en un inicio y los aspectos que superaron.

\section{1) Dificultades superadas por el grupo de control}

De manera general hubo mejoras tanto en el GC como en el GE al resolver el cuestionario final, después de un proceso de enseñanza que duró un mes, teniendo distintos acercamientos cada grupo. En este apartado se mencionan las mejoras de cada uno de los grupos.

En el grupo de control (GC), se encontró que hubo un avance en el tema de optimización pues su porcentaje de respuestas correctas tuvo un incremento de más del doble en ambas tareas (problema 3 y problema 5), lo que se debió preferentemente a que los estudiantes emplearon correctamente el criterio de la primera derivada para la obtención de la mínima área que es la que se solicitaba. Se observó que estos estudiantes emplearon más la memoria al seguir cada uno de los pasos para la obtención de los puntos críticos.

Otro tema en el que hubo mejoría por parte del GC fue el de diferenciales, ya que en el cuestionario diagnóstico sólo el 3\% de los alumnos resolvió correctamente el problema de

diferenciales y en el cuestionario final aumentó al 18\%. Los alumnos argumentaron que el tema de diferenciales no lo habían trabajado en el nivel medio superior y tampoco en su primer semestre de cálculo, o al menos no habían resuelto problemas de diferenciales, solo conocían algo de la teoría, pero después de las clases dadas, entre el cuestionario inicial y el final, ya comprendieron en lo que se puede aplicar este tema. 
Estudio sobre las dificultades que tienen estudiantes de nivel superior en la resolución de problemas de cálculo, y empleo de un Escenario de aprendizaje tecnopedagógico como apoyo en el trabajo del aula

\section{Análisis de resultados del grupo experimental}

En relación al grupo experimental (GE) los resultados obtenidos en el cuestionario final en comparación con los del cuestionario diagnóstico, se muestran en la gráfica de la Fig. 8. En donde se observa que los estudiantes tuvieron una notable mejoría en los temas de optimización, seguido del tema de razones de cambio relacionadas, diferencial de una función y obtención de puntos críticos y comportamiento de una función.

\section{1) Dificultades superadas por el GE}

En el grupo experimental (GE) Alrededor del $40 \%$ de los estudiantes, al principio emplearon la estrategia de "ensayo y error", al dar valores para determinar una aproximación cercana a las dimensiones de la hoja donde se encuentra el póster, lo que significa que estos estudiantes le dieron sentido a la situación planteada en el problema (se ve reflejado el trabajo en clase al usar esta estrategia empleando las simulaciones mostradas), como segunda opción plantearon la función que representaba el área que se iba a minimizar y trabajaron con el criterio de la segunda derivada para concluir que el valor sustituido representaba un mínimo.

En el problema 5, que también era de optimización, algunos alumnos al principio tuvieron dificultad en plantear la función que expresaba algebraicamente la situación, pues se incluían dos semicírculos, la confusión radicó en que trabajaron con dos círculos en lugar de con dos semicírculos. Pero el uso de la estrategia de ensayo y error les ayudó a llegar a la función correcta. Posteriormente emplearon el criterio de la primera y segunda derivada para darle solución.

El porcentaje de incremento en la resolución de los problemas de diferenciales cambió del $3 \%$ al $42 \%$, lo que equivale a decir que una catorceava parte de los estudiantes resolvió correctamente el problema en el cuestionario inicial, mientras que en el final aumentó catorce veces la cantidad de estudiantes, lo que se debió a las estrategias de aprendizaje y a los recursos didácticos empleados como parte del Escenario de Aprendizaje Tecnopedagógico (EATP), pues los estudiantes observaban en las simulaciones el cambio que se generaba en la figura de la cual se solicitaba determinar el error de medición. Los estudiantes reconocieron al error como la diferencial de la variable independiente y al error del volumen como la diferencial de la función.

En relación al problema de razones de cambio relacionadas, el porcentaje de estudiantes que logró resolver de forma correcta el problema cambió de 18.2\% que obtuvo en el cuestionario 
inicial, a 63.2\% alcanzado en el cuestionario final (más del triple), el éxito se debió al tipo de estrategias empleadas, como esquematizar el problema, colocando las variables identificadas en el texto, así como estableciendo las relaciones adecuadas para obtener una función expresada en términos de una sola variable.

En la Tabla 9 se muestran los resultados finales de ambos grupos, donde se aprecia el efecto del trabajo realizado con el escenario de aprendizaje tecnopedagógico.

Tabla 9. Diferencia en los resultados finales de los dos grupos.

\begin{tabular}{|c|c|c|c|}
\hline Análisis de los datos & Grupos & & \\
\hline Columna D & GE_F & & \\
\hline Vs. & Vs. & & \\
\hline Columna B & GC_F & & \\
\hline \multicolumn{4}{|l|}{ Prueba t no pareada con corrección de Welch } \\
\hline P valor & $<0.0001$ & & \\
\hline P resumen del valor & $* * * *$ & & \\
\hline ¿Significativamente diferentes? $(P<0.05)$ & Yes & & \\
\hline ¿Valor P de una o dos colas? & Dos colas & & \\
\hline T corregida de Welch, df & $\mathrm{t}=6.394 \mathrm{df}=57.83$ & & \\
\hline \multicolumn{4}{|l|}{ ¿Qué tan grande es la diferencia? } \\
\hline Mean \pm SEM of column B & $4.188 \pm 0.2436, n=32$ & & \\
\hline Mean \pm SEM of column D & $6.848 \pm 0.3374, n=33$ & & \\
\hline Diferencia entre los medios & $2.661 \pm 0.4162$ & & \\
\hline $95 \%$ intervalo de confianza & 1.828 to 3.494 & & \\
\hline R cuadrada & 0.4141 & & \\
\hline \multicolumn{4}{|l|}{ F test para comparar varianza } \\
\hline F,DFn, Dfd & $1.979,32,31$ & & \\
\hline $\mathrm{P}$ valor & 0.0606 & & \\
\hline P resumen del valor & $\mathrm{ns}$ & & \\
\hline ¿Significativamente diferentes? $(P<0.05)$ & No & & \\
\hline Modelo de comparación & SS & DF & Probability it is correct \\
\hline Hipótesis Nula: Idénticas poblaciones de medios & 294.2 & 64 & $0.00 \%$ \\
\hline $\begin{array}{l}\text { Hipótesis Alternativa : Distintas poblaciones de } \\
\text { medios }\end{array}$ & 179.1 & 63 & $100.00 \%$ \\
\hline Razón de probabilidades & & & 0.0 \\
\hline Diferencia en AICc & & & 30.04 \\
\hline
\end{tabular}

Al hacer una comparativa entre el grupo de control y el experimental se observa en la Tabla 10 que el GE obtuvo más respuestas correctas en general que el GC. 
Estudio sobre las dificultades que tienen estudiantes de nivel superior en la resolución de problemas de cálculo, y empleo de un Escenario de aprendizaje tecnopedagógico como apoyo en el trabajo del aula

Tabla 10. Diferencias entre estado inicial y estado final para cada grupo.

\begin{tabular}{|c|c|c|c|}
\hline Análisis de los datos & Grupos & & \\
\hline Columna B & GC_F & & \\
\hline Vs. & Vs. & & \\
\hline Columna A & GC_I & & \\
\hline \multicolumn{4}{|l|}{ Prueba t no pareada con corrección de Welch } \\
\hline P valor & $<0.0001$ & & \\
\hline$P$ resumen del valor & $* * * *$ & & \\
\hline ¿Significativamente diferente? $(\mathrm{P}<0.05)$ & Yes & & \\
\hline ¿Valor $P$ de una o dos colas? & Dos-colas & & \\
\hline T corregida de Welch, df & $\mathrm{t}=4.948 \mathrm{df}=63.56$ & & \\
\hline \multicolumn{4}{|l|}{ ¿Qué tan grande es la diferencia? } \\
\hline Mean \pm SEM of column A & $2.286 \pm 0.2972, n=35$ & & \\
\hline Mean \pm SEM of column B & $4.188 \pm 0.2436, n=32$ & & \\
\hline Diferencia entre los medios & $1.902 \pm 0.3843$ & & \\
\hline $95 \%$ intervalo de confianza & 1.134 to 2.670 & & \\
\hline R cuadrada & 0.2781 & & \\
\hline \multicolumn{4}{|l|}{ F test para comparar varianza } \\
\hline F,DFn, Dfd & $1.628,34,31$ & & \\
\hline P valor & 0.1737 & & \\
\hline P resumen del valor & ns & & \\
\hline ¿Significativamente diferentes? $(\mathrm{P}<0.05)$ & No & & \\
\hline Modelo de comparación & SS & $\mathrm{DF}$ & Probability it is correct \\
\hline Hipótesis Nula: Idénticas poblaciones de medios & 224.5 & 66 & $0.01 \%$ \\
\hline $\begin{array}{l}\text { Hipótesis Alternativa: Distintas poblaciones de } \\
\text { medios }\end{array}$ & 164.0 & 65 & $99.99 \%$ \\
\hline Razón de probabilidades & & & 0.0 \\
\hline Diferencia en AICc & & & 18.83 \\
\hline
\end{tabular}

\section{Discusión o Conclusiones}

Sobre lo que se encontró al realizar el análisis a las respuestas dadas por los estudiantes en el grupo experimental y al contrastarlo con lo que señalan los autores de los aspectos teóricos en relación a la visualización y al trabajo de la aritmética para poder llegar a un trabajo algebraico, se tiene lo siguiente. 
Después de haber trabajado en el grupo experimental con el modelo TPCAK, se obtuvieron buenos resultados ya que el promedio del grupo aumentó, de 2.7 a 6.3 , lo que implica que hubo un mejor rendimiento en este grupo durante el mes de trabajo. La mejoría se atribuye a las diferentes estrategias que emplearon en la resolución de los distintos problemas, predominando la estrategia de "ensayo y error" como primera opción al dar algunos valores que les permitía tener un panorama sobre el aumento o decremento de la situación objeto de que se tratara, es decir los estudiantes pudieron trabajar con la aritmética para visualizar casos particulares para después trabajar con generalizaciones, lo que se asemeja a lo mencionando por Kaput (2000, 2008); Kieran (2007) y Artigue (2011). También la estrategia de "esquematizar el problema" y ubicar las variables reconociendo a las independientes y a las dependientes, mediante las cuales llegaban a determinar la función que representaba el problema y, como parte de esta misma estrategia, el expresar a la función en términos de una sola variable y después determinar una ecuación auxiliar para despejar la variable requerida, para posteriormente, mediante el uso de algún criterio, como el de la primera derivada o segunda, en el caso de problemas de optimización, o la derivación implícita con respecto al tiempo, en el caso de razones de cambio relacionadas, los estudiantes llegaban a obtener las respuestas a los planteamientos realizados. Este trabajo desarrollado por los estudiantes alude a lo señalado por Hitt (2014), Sánchez, García \& Llinares (2008), Oliveros (1999), Duval (1998), en relación empleo de diferentes representaciones, no solo el algebraico, sino el icónico, el numérico y el tabular.

En el grupo de control (GC), no hubo mejora en el rendimiento académico pues su promedio cambió de 2.6 a 3.6, lo que se debió al tipo de estrategias empleadas por los estudiantes, las cuales se recostaron en el uso de fórmulas usadas durante la clase pero sin haber adquirido un sentido para los alumnos ya que fue un aprendizaje de carácter memorístico.

Se concluye que el escenario Tecnopedagógico constituye un apoyo para que el estudiante maneje con más soltura y previo entendimiento, las fórmulas implicadas en algunas estrategias de resolución de problemas. La integración de la tecnología productiva en la enseñanza necesita considerar los tres aspectos (Contenido, pedagogía y tecnología) no de forma aislada, sino dentro del complejo de relaciones en el sistema definidas por los tres elementos clave 
Estudio sobre las dificultades que tienen estudiantes de nivel superior en la resolución de problemas de cálculo, y empleo de un Escenario de aprendizaje tecnopedagógico como apoyo en el trabajo del aula

\section{$\underline{\text { Referencias }}$}

Area, M. \& Adell, J. (2009). E-Learning: Enseñar y aprender en espacios virtuales. En Tecnología Educativa. La formación del profesorado en la era de Internet, editado por De Pablos J. (391-424).

Artigue, Michelle. (2011). Tecnología y enseñanza de las matemáticas: desarrollo y aportes de la aproximación instrumental. Cuadernos de Investigación y Formación en Educación Matemática, 8 (1): 13 - 33 .

Boneu, Josep M. (2007). Plataformas abiertas de e-learning para el soporte de contenidos educativos abiertos. RUSC Universities and Knowledge Society Journal, 4(1): 36-47.

Cuesta, A; Escalante, J.; Ruiz J. (2016). Velocidad. Significados manifestados por estudiantes universitarios a partir de representaciones gráficas. Avances de Investigación en Educación Matemática, (9): 105 - 125.

Drijvers, P., Kieran, C., Mariotti, M.-A., Ainley, J., Andresen, M., Chan, Y., Meagher, M. (2009). Integrating Technology into Mathematics Education: Theoretical Perspectives. En Mathematics Education and Technology-Rethinking the Terrain: The 17th ICMI Study, editado por C. Hoyles (89-132). Boston, MA: Springer US. doi:https://doi.org/10.1007/978-1-4419-0146-0_7

Duval, Raymond (1988). Registros de Representación Semiótica y funcionamiento cognitivo del pensamiento. Investigaciones en Matemática Educativa II: 173-201.

Flores, A. (2013). Ayudando a futuros profesores a mejorar la comprensión conceptual del cálculo. En La enseñanza del cálculo Diferencial e Integral compendio de investigaciones y reflexiones para profesores, formadores e investigadores en matemática educativa, editado por Cuevas, Armando, Pluvinage, Francoise \& Flores, Attelier (43 - 83). México: Pearson.

García-Peñalvo, F. J. (2005). Estado actual de los sistemas E-Learning. Teoría de la Educación. Educación y Cultura en la Sociedad de la Información, 6(2): 1.

Harris, J. and Hofer, M. (2009). Instructional planning activity types as vehicles for curriculumbased TPACK development, En Research highlights in technology and teacher education, Chesapeake, Society for Information Technology in Teacher Education (SITE)., Editado por Cleborne D. Disponible en: 
http://activitytypes.wmwikis.net/file/view/HarrisHoferTPACKDevelopment.pdf Acceso en: 8/10/2018.

Hernández-Sampieri, R., Fernández-Collado, C. \& Baptista-Lucio, P. (2014). Metodología de la Investigación. $6^{\mathrm{a}}$. Ed. México: McGraw-Hill.

Hitt, Fernando. (2013). Un análisis sobre la enseñanza del concepto de derivada en el nivel preuniversitario del rol del libro de texto y del uso de sus conexiones con la tecnología. En La enseñanza del cálculo Diferencial e Integral, editado por Cuevas Armando \& Pluvinage, Fancoise. Pearson. México.

Hitt, Fernando. (2014). Nuevas Tendencias en la Enseñanza del Cálculo: La Derivada en Ambientes TICE. AMIUTEM, 2(2): 1-19, Disponible en:

http://revista.amiutem.edu.mx/ojs/index.php/relecamiutem/article/view/20 Acceso en: $21 / 11 / 2018$.

Hitt, F., González-Martín, A. Et Morasse C. (2008). Visualization and students' functional representations in the construction of mathematical concepts. An example: The concept of co-variation as a prelude to the concept of function. In 11th International Congress on Mathematics Education (ICME11), Topic Study Group 20 (TSG 20), Visualization in the Teaching and Learning of Mathematics, Monterrey, N. L., Mexico. http://tsg.icme11.org/tsg/show/21

INEGI (2018). Encuesta Nacional sobre Disponibilidad y Uso de Tecnologías de la Información en los Hogares 2018. Disponible en:

http://www.beta.inegi.org.mx/proyectos/enchogares/regulares/dutih/2018/default.htm._ Acceso en: $12 / 10 / 2018$

IPN. ESCOM. Plan de estudios de Cálculo Aplicado. 2009. Recuperado de: http://www.escom.ipn.mx/docs/ofertaEducativa/uapdf/calculoAplicado.pdf

Kaput, J.J. (2008). What is algebra? What is Algebraic Reasoning? In J.J. Kaput, D. Carraher \& M.L.Blanton (Eds.), Algebra in the Early Grades (pp. 5-17). New York: Routledge.

Kieran, C. (2007). Learning and Teaching Algebra at the Middle School Through College Levels. En Lester, F. K. (Ed.). Second Handbook of Research on Mathematics Teaching and Learning (pp. 707-762). Reston, Virginia: NCTM e IAP.

Koehler, M. and Mishra, P. (2006). Technological Pedagogical Content Knowledge: A Framework for Teacher Knowledge. Teachers College Record, 108(6): 1017-1054. 
Estudio sobre las dificultades que tienen estudiantes de nivel superior en la resolución de problemas de cálculo, y empleo de un Escenario de aprendizaje tecnopedagógico como apoyo en el trabajo del aula

Disponible en inglés en:

http://punya.educ.msu.edu/publications/journal_articles/mishra-koehlertcr2006.pdf Acceso en: $15 / 11 / 2018$.

Moschkovich, J. N. y Brenner, M. (2000). Integrating a Naturalistic Paradigm Into Research on Mathematics and Science Cognition and Learning. En R. Lesh y A. Kelly (Eds.), Handbook of Research Design in Mathematics and Science Education (pp. 457-486). Mahwah, NJ: Lawrence Erlbaum Associates.

Sánchez, G., García, M., \& Llinares, S. (2008). La Comprensión de la Derivada como Objeto de Investigación en Didáctica de la Matemática. Revista Latinoamericana de Investigación en Matemática Educativa, 11(2): 267 - 296.

Stewart, J. (2012). Cálculo de una variable. Trascendentes tempranas. Trad. de Rodríguez Pedroza, M. C. (2012). México: Cengage Learning Editores.

Oliveros, J. R. (1999). El estudio de la Tasa de Cambio Instantánea en el entendimiento de la Derivada Situado en el salón de Clase. 1999. Tesis Doctorado en Ciencias especialidad de matemática educativa. Cinvestav, México.

Larson, R \& Edwars, B. H. (2010). Cálculo 1 en una variable. 9a. Ed. México: McGraw -Hill

Rosenberg, M. J. (2001). E-Learning. Strategies for delivering knowledge in the Digital Age. New Cork, McGraw-Hill.

Saboya, M. (2010). Élaboration et analyse d'une intervention didactique co-construite entre chercheur et enseignant, visant le développement d'un contrôle sur l'activité mathématique chez les élèves du secondaire. Tesis de doctorado no publicada. Université du Québec à Montréal.

Shulman, L. S. (2005).Those who understand: Knowledge growth in teaching. Educational Researcher, v. 15, n. 2, 4-14. 1986. Trad. y edición española ("El saber y entender de la profesión docente") en Estudios Públicos (Centro de Estudios Públicos, Chile), (99):195224

Swan, K., Kratcoski, A., And Van't Hooft, M. (2007). Highly Mobile Devices, Pedagogical Possibilities, and How Teaching Needs to Be. Educational Technology, 47(10): Disponible en: http://www.rcet.org/research/publications/ET_May-June_2007_swan.pdf Acceso en: 14/10/2018

UNESCO. (2013). Directrices para las políticas de aprendizaje móvil. UNESCO: Francia. 
Zimmermann, W. \& Cunningham, S. (Eds.) (1991). Visualization in Teaching and Mathematics (pp. 25-37). MAA Series, No. 19. USA. 